[Institut für Plasmaphysik Association EURATOM-KFA

Wall Conditioning in Fusion Devices and its Influence on Plasma Performance

J. Winter 


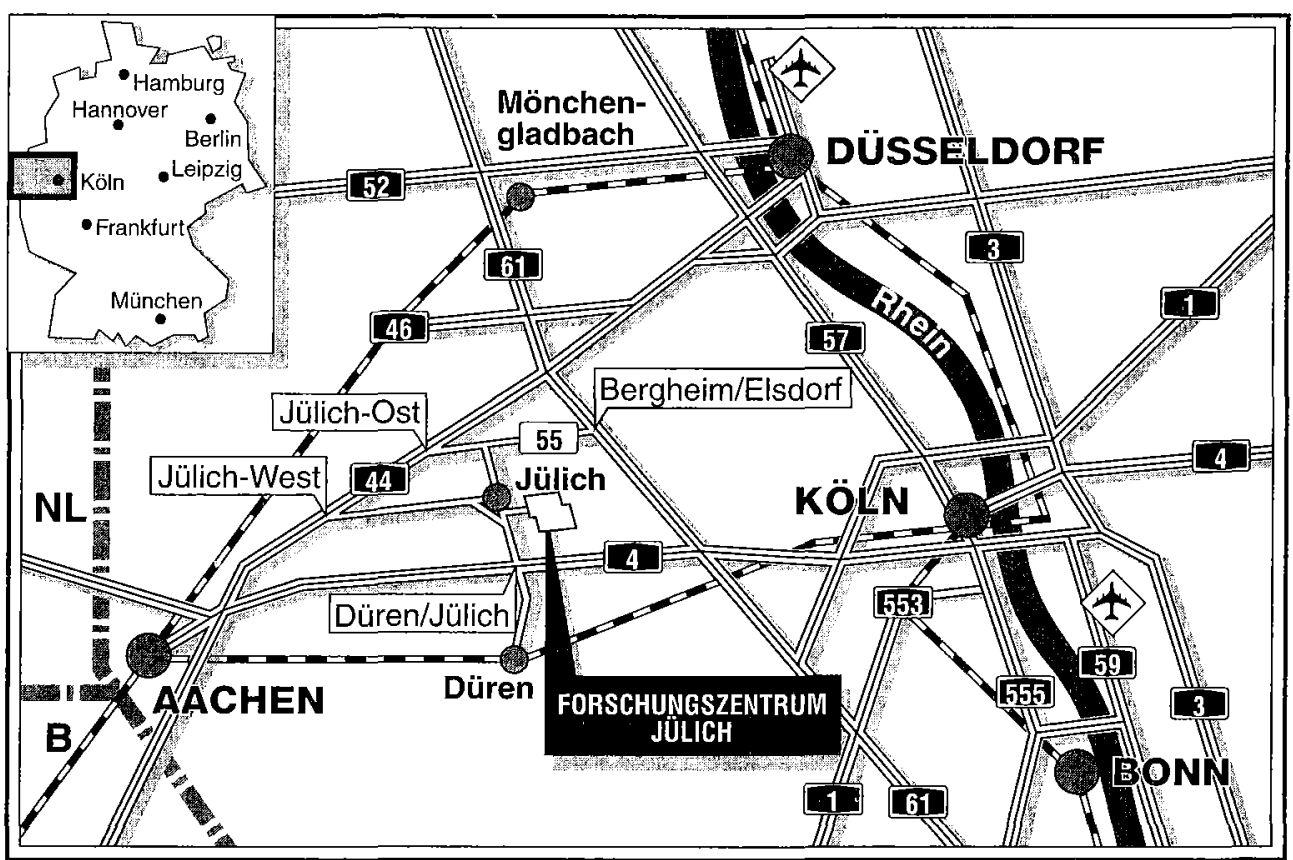

Berichte des Forschungszentrums Jülich ; 3217

ISSN 0944-2952

Institut für Plasmaphysik Jül-3217

Association EURATOM-KFA

Zu beziehen durch: Forschungszentrum Jülich $\mathrm{GmbH}$ - Zentralbibliothek D-52425 Jülich - Bundesrepublik Deutschland

Telefon: 02461/61-6102 Telefax: 02461/61-6103 - Telex: 833556-70 kfa d 


\title{
Wall Conditioning in Fusion Devices and its Influence on Plasma Performance
}

\author{
J. Winter
}


$1 . \quad$ Introduction

2. The Role of Plasma Impurities

3. Oxygen Impurities

4. Hydrogen Recycling

5. Methods of Wall Conditioning

5.1 Discharge Cleaning in Hydrogen and in other Reactive Gas Mixtures

5.2 Glow Cleaning in Helium and Disruptive Discharge Cleaning

5.3 Metal Film Gettering

5.3.1 Ti- and $\mathrm{Cr}$-Gettering

5.3.2 Be-Gettering

5.4 Plasma Assisted Deposition of Thin Films

(Carbonization, Boronization, Siliconization)

5.5 Film Deposition during Tokamak Discharges

6. Influence of Wall Conditioning on Tokamak Discharges

6.1 Discharge Cleaning in Hydrogen and Helium

6.2 Medium $\mathrm{Z}$ Gettering: ( $\mathrm{Ti}$ and $\mathrm{Cr}$ )

6.2.1 Ti-Gettering

6.2.2 Cr-Gettering

6.3 Plasma Assisted Thin Film Deposition and Be Evaporation

6.3.1 Carbonization

6.3.2 Boronization, Siliconization and Be-Gettering

6.3.2.1 Influence on Plasma Impurities and Density Limits

6.3.2.2 Influence on Hydrogen Recycling

6.3.3 Lifetime of Coatings

$6.4 \quad$ Li-Pellet Injection

7. Wall Conditioning in Future Devices

8. Conclusions and Outlook 



\section{Introduction}

In magnetically confined fusion plasmas a strong nonlinear connection exists between the processes occurring at the wall, the properties of the plasma edge, and those of the main plasma. The ion- and electron temperature profiles, the power flow in the plasma edge and its radiated fraction (radiative edge), the concentration and penetration depth of neutral hydrogen atoms and of plasma impurities into the scrape-off-layer (SOL) are examples for such strongly interlinked quantities. Via complex mechanisms they modify energy and particle transport, plasma confinement, and the performance of the discharge. High performance implies maximizing the fusion triple product $n_{i} T_{i} T_{E}$ of deuteron and triton density $n_{i}$, ion temperature $T_{i}$ and energy confinement time $T_{E}$ in a thermal plasma. Hence it is important to achieve high densities, avoid the cooling of the plasma by excessive radiation of power, avoid dilution of the triton and deuterons by impurities, and to promote high energy confinement, consistent with the requirements of energy removal and helium pumping.

The appropriate architecture of the plasma edge appears to be essential in order to obtain thermonuclear fusion plasmas with high performance. The edge plasma itself is strongly influenced by the plasma-wall interaction. These plasma-surface interaction processes have been reviewed e.g. by McCracken and Stott [1]. Detailed descriptions of various mechanisms and diagnostic aspects can be found in ref. [2]. Recent reviews of plasma edge phenomena have been published by Stangeby and McCracken [3], and by various authors in ref. [4].

Atoms and ions from the plasma have a strong interaction with matter. Their range is limited to typically several $10 \mathrm{~nm}$ from the surface even in low- $Z$ target materials (Be or $\mathrm{C}$ ) with small stopping powers. It is this thin layer, the near-surface region, where plasma-surface interactions take place and where all the basic processes like sputtering, chemical erosion, trapping, reflection etc. occur. These processes may induce the release of wall material and thus introduce plasma impurities or have an effect on the hydrogenic particle balance (hydrogen recycling). It is obvious, that a modification of this near surface region e.g. by discharge cleaning or by depositing thin films of appropriate chemical composition and physical structure will bring about major changes in the plasma-surface-interaction processes and may thus allow an active control of phenomena critical to the plasma and its performance.

Although only partially understood today, plausible interrelations of plasmasurface interactions and different high confinement modes from the point of view of plasma theory were discussed in a recent review paper by 
S.l. Itoh et al. [5]. One of the best investigated examples, the $\mathrm{H}$-mode, is characterized by pedestals in the density and temperature profiles. It is widely accepted that a strongly sheared radial electric field at the edge and the associated sheared poloidal rotation of the plasma lead to a suppression of turbulence in the edge region. This induces the formation of a transport barrier, manifest by the strong edge gradients in plasma density and temperature.

It is argued [5] that a low rate of charge exchange reactions in the edge due to a small number of recycling neutral deuterium atoms is important to minimize a loss of momentum and to avoid elimination of the radial electric field. The penetration depth of the neutrals should be as small as possible.

Measurements in TEXTOR have shown that the high energy fraction of reflected neutral atoms is smaller for a limiter of low $Z$ than of high $Z$ materials [6]. This can be understood from the dependence of the energy transfer on the mass ratio in collisions of projectile and substrate atoms. Thus, choosing low $Z$ wall elements appears to be favorable to minimize the penetration of reflected neutrals.

Theory indicates [5] that the bifurcation to the H-regime is eased by low values of radial ion fluxes driven by collisional processes. A low value of $Z_{\text {eff }}$ is thus favorable, and low impurity levels should be aimed for. In view of discharges with a radiative boundary and high confinement (TEXTOR I-mode [7] $\mathrm{CDH}$ - and CDL-Mode in ASDEX-Upgrade [8]), this condition can be formulated more generally: the control of impurity species and of their fluxes is important.

As suggested for the $\mathrm{H}$-mode, but probably applying to other high confinement regimes also, important ingredients in the recipes for achieving high confinement regimes appear to be: low neutral hydrogen influxes, controlled impurity influxes (concerning both the species and the rates), and low $Z$ wall materials.

This review will give an overview of wall conditioning techniques for the active control of two critical elements for high performance operation: the plasma impurities and the hydrogen recycling. The intervening mechanisms will be discussed and illustrated by examples from fusion devices. 


\section{The Role of Plasma Impurities}

Plasma impurities cause two main effects related to high performance: radiation of power, and dilution of the hydrogenic species. Furthermore they can influence collision processes via $Z_{\text {eff }}$ and the plasma resistivity and hence impact plasma stability and global energy confinement.

The radiated power by line radiation increases strongly with $Z$ as shown in fig. 1 . The energy loss by Bremsstrahlung is proportional to $Z^{2}$. Cooling of peripheral plasma zones by radiation may be a desired effect in order to reduce the convective power flow to the limiters or divertor plates of a tokamak along magnetic field lines and distribute it over a large surface area. According to their atomic number $Z$ and their various ionization and excitation potentials, different impurity atoms will radiate in different plasma regions according to the electron temperature profile $T_{e}(r)$. Under the simplifying assumptions of coronal equilibrium, the radiation from a transition with ionization potential $E_{i}$ occurs from a plasma shell located at $r$ with $T_{e}(r)$ $\sim 1 / 3 E_{i}$. As has been shown e.g. in TEXTOR, enhanced edge radiation can significantly improve energy confinement [7] which makes the concept of a highly radiative plasma boundary attractive for ITER and reactors. Care must be taken, however, that the radiation does not cool the hot plasma core and that the dilution remains small. The correct impurity species has to be selected. In TEXTOR, as an example, neon $(Z=10)$ [7] and silicon $(Z=14)$ $[7,9]$ have been used successfully.

Plasma impurities also lead to a dilution of the hydrogenic fuel. Electrons and the nuclei of impurity atoms take the place of those from deuterons and tritons. Assuming $n_{D}=n_{T}=n$ and $n_{e}=2 n$ the fusion power density $p_{a}$ is $\propto n^{2}$. At full ionization and at constant $n_{e}$, the concentration $c_{z}=$ $\mathrm{n}_{\mathrm{z}} / 2 \mathrm{n}$ of impurity atoms with atomic number $\mathrm{Z}$ leads to decreased fusion power $\mathrm{p}_{a}{ }^{\prime}$ :

$$
\left.\mathrm{p}_{a}^{\prime}=\mathrm{p}_{a}\left[1-\mathrm{Z} \cdot \mathrm{c}_{\mathrm{z}}\right)\right]^{2} \text {. }
$$

For $\mathrm{c}_{\mathrm{z}}=3 \%$, this reduction is $12 \%$ for $\mathrm{He}, 23 \%$ for $\mathrm{Be}, 28 \%$ for $\mathrm{B}$ and $33 \%$ for $C$. The dilution cannot be counteracted by simply increasing $n_{D}$ and $n_{T}$. In a given device the maximum achievable density is limited due to the critical plasma pressure $n_{e} T_{e}+\left(n_{D}+n_{T}+n_{z}\right) T_{i}$ (B-limit).

Plasmas once contaminated tend to be further polluted at an enhanced rate. If plasma impurities return to the wall, they are usually multiply ionized (charge q). Upon approach to the surface, they are accelerated in the sheath potential $U_{s} \sim 3.8 \mathrm{kT}_{\mathrm{e}}[3]$ up to a total impact energy $E$ of

$$
\mathrm{E} \sim \mathrm{qU}_{\mathrm{s}}+2 \mathrm{kT} \mathrm{T}_{\mathrm{i}} \text {. }
$$


These multiply charged impurity ions will lead to enhanced erosion by sputtering. In the case of TEXTOR, the mean charge state of carbon ions hitting the limiter is $q=4$ [10]. For a typical value of $T_{e}=25 \mathrm{eV}$ at the limiter, the additional energy gain in the sheath is $360 \mathrm{eV}$. This leads to an increase of the carbon self sputter yield on graphite wall components from about $1 \times 10^{-2}$ to $2 \times 10^{-1}$, for example.

From these basic consideration one concludes that the impurity release has to be reduced to a low level from the very beginning of the discharge. The tolerable amount depends on the type of impurity atoms. The value of $Z$ and the sticking probability to the surface are important as is their shielding from penetration through the SOL. The steady state concentration of impurity atoms in the plasma is determined by the balance between influx and outflux rates. The latter is inversely proportional to the particle containment time $\tau_{p}$ of the species which is intimately coupled to the transport properties. Therefore, an evaluation of acceptable primary impurity release rates and influx rates has to take the transport properties of the respective plasma regime into account. In general, good particle confinement requires small primary erosion rates.

\section{Oxygen Impurities}

It has been recognized early in nuclear fusion research that oxygen plays a particular role in the plasma impurity generation [1]. Two alternative ways of controlling the oxygen content of the plasma have been explored: hydrogen discharge cleaning, optimized to yield a maximum of oxygen removal out of the vessel, and application of gettering techniques to bind oxygen strongly to the surface.

The ability of a material to getter (bind) oxygen plasma impurities may be assessed in a first approximation by its oxygen affinity and by the desorption temperatures and desorption rates of the oxides. The oxygen affinity can be expressed in terms of the formation enthalpy of the oxides, normalized to the number of oxygen atoms $\Delta G / O$ (as listed in table 1 ). According to this figure of merit, Beryllium $(Z=4)$ and Boron $(Z=5)$ and $S i(Z=14)$ are good low- $Z$ getters. Lithium has a low binding energy, which is less than that of water, and is thus not expected to be a good oxygen getter in the presence of atomic hydrogen. Titanium and Chromium are good medium $\mathrm{Z}$ getter materials.

Oxygen plays a special role in devices with graphite covered walls. Upon impact of energetic oxygen ions onto a pure carbon surface, oxygen is initially retained up to a concentration of about 0.25 oxygen per carbon atom 
within the implantation zone, but thereafter it is reemitted in the form of CO and $\mathrm{CO}_{2}$ with a yield close to unity even at room temperature--[11]. Measurements have shown that these molecules are not all released at thermal energies but reveal a fast component of about $0.25 \mathrm{eV}$ [12]. This may lead to a high penetration probability into the main plasma. After dissociation and ionization, $\mathrm{CO}$ and $\mathrm{CO}_{2}$ are sources for both carbon and oxygen plasma impurities. The yield of almost unity for $\mathrm{CO}$ and $\mathrm{CO}_{2}$ formation by oxygen ions impinging on carbon and their volatililty imply that oxygen recycles with a recycling coefficient approaching unity in a tokamak with carbon walls.

The recycling behavior of oxygen in an all carbon machine has been measured in the carbonized TEXTOR. Figure 2 shows the oxygen OVI light emission as a function of the discharge number. Between discharge 494 and 495 a glow discharge in helium of 15 min duration was made, desorbing oxygen as $\mathrm{CO}$ from the near surface layer and removing it out of the vessel (see below). Subsequent discharges have a lower oxygen content which gradually returns to the previous level, however. The source of oxygen is likely to be water vapor outgassing from surfaces shielded from the conditioning plasmas. A schematic representation of the oxygen cycle is shown in figure 3 [13].

Boron containing carbon materials retain (getter) significantly more oxygen than pure carbon does. Their retention is increasing with the boron content. $\mathrm{CO}$ and $\mathrm{CO}_{2}$ reemission from these materials occurs at significantly lower yields than in graphite and at higher desorption temperatures. The reduction of the yield depends on the boron concentration. It is of the order of 5-8 for boroncarbide i.e. for $B / C=4$, and for boronization films (see below) of similar composition (see fig. 4). The reemission oxides from different $B / C$ compounds during oxygen ion exposure has been investigated [11]. The most prominent boron oxide species is $\mathrm{BO}$. The maximum of the desorption rate is observed around $1000-1400{ }^{\circ} \mathrm{C}$.

Titanium and chromium oxides have a low volatility and are expected to be stable up to temperatures in excess of $1300^{\circ} \mathrm{C}$. No data are known to the author on the desorption of these oxide from e.g. Ti or $\mathrm{Cr}$ covered graphite surfaces which are irradiated with oxygen ions. Data are also lacking for the release rates of beryllium oxides and beryllium hydroxides on carbon. It has been reported that, at temperatures above about $280^{\circ} \mathrm{C}$, beryllium metal diffuses trough a surface oxide layer and thus replenishes the surface with active beryllium atoms [14].

The formation of non stoichiometric hydroxides and radicals is likely under the non equilibrium conditions of a plasma-exposed tokamak surface. They 
can have vastly differing volatilities and it is thus difficult to give a simple figure of merit for their release.

Nickel and Iron, the main constituents of stainless steel and Inconel, are poor getters. Their oxides are readily reduced by a hydrogen plasma. The atomic hydrogen species react on these surfaces to produce water vapor. Water vapor desorbs from the surfaces and becomes ionized in the boundary plasma and is thus a vehicle to introduce $O$ impurity atoms into the plasma. As has been suggested by Dietz and Waelbroeck [15] and measured in a tokamak by Waelbroeck et al. [16], water vapor is the primary source for oxygen impurities in a fusion device with metallic walls. On the other hand, this property can be used advantageously in discharge cleaning techniques.

To understand the special role of water cycling between plasma and wall surface, it is important to consider the equilibrium of the hydrolyzation reactions of metal oxides $(\mathrm{MO})$ on the wall with atomic hydrogen $(H)$ leaving the plasma:

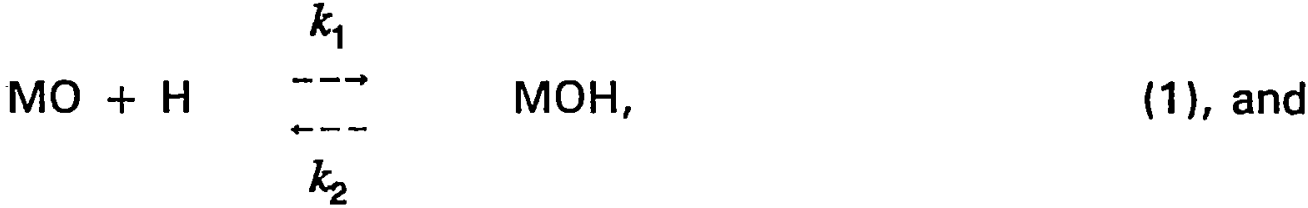

$$
\begin{aligned}
& \mathrm{MOH}+\mathrm{H} \stackrel{k_{3}}{\rightarrow} \quad \mathrm{M}+\mathrm{H}_{2} \mathrm{O}
\end{aligned}
$$

The reaction rate constants $k_{1}, k_{2}, k_{3}$ have not been measured for the metaloxides of vessel walls ( $\mathrm{FeO}, \mathrm{Cr}_{2} \mathrm{O}_{3}$, etc). The trend of the reactions may however be derived from the known equilibrium constants $K_{p}=p\left(H_{2} \mathrm{O}\right)$ / $\mathrm{p}\left(\mathrm{H}_{2}\right)$ for the reduction of these oxides with molecular hydrogen:

$$
\mathrm{MO}+\mathrm{H}_{2} \stackrel{K_{p}}{\rightarrow} \quad \mathrm{M}+\mathrm{H}_{2} \mathrm{O}
$$

where $p\left(\mathrm{H}_{2} \mathrm{O}\right)$ and $\mathrm{p}\left(\mathrm{H}_{2}\right)$ denote the partial pressures of $\mathrm{H}_{2} \mathrm{O}$ and $\mathrm{H}_{2}$ over the metaloxide. Figure 5 shows a plot of $\mathrm{K}_{\mathrm{p}}$ for various oxides as a function of temperature. As can be seen, $\mathrm{FeO}$ is much easier reduced than $\mathrm{TiO}^{\circ}$ or $\mathrm{Cr}_{2} \mathrm{O}_{3}$. Although clean Fe surfaces may act as a getter for oxygen, $\mathrm{H}_{2} \mathrm{O}$ vapor is formed easily and recycled. NiO, not shown in the graph, the most prominent oxide on Inconels, is reduced even more easily. 


\section{Hydrogen Recycling}

Hydrogen recycling, the repeated interchange of hydrogen species between the plasma and the wall, is a complex process. Its appearance is different on metallic surfaces and on ceramics like graphite or on boron and silicon containing layers.

The hydrogen recycling in a metal environment (non hydride formers) has been analyzed in various publications and is relatively well understood [17 20]. The behavior is characterized by a flux dependent dynamic wall pumping, the rate of which decreases during plasma exposure. The recycling flux is determined by the value of the recycling constant $R=D / K_{r}$, where $D$ is the hydrogen diffusivity in the bulk metal and $K_{r}$ is the recombination rate constant for the recombination of hydrogen atoms into hydrogen molecules at the surface. $R$ can be measured by analyzing the pressure response function in a vessel the wall of which is suddenly exposed to a hydrogen plasma or in which a hydrogen plasma is suddenly switched off ("Wall Pump and Release Effect") [19].

At the beginning of the plasma exposure a subsurface hydrogen concentration is built up. The wall strongly absorbs hydrogen atoms in this phase. As the concentration increases, surface recombination into molecules increases proportionally to the square of the concentration and hydrogen is progressively released back to the plasma: the wall pumping decreases. After the characteristic recycling time $\tau_{\mathrm{R}}=\mathrm{R} / \Phi, 50 \%$ of the initially pumped hydrogen flux is reemitted back from the surface $(\Phi$ is the impinging hydrogen flux density). Some hydrogen also permeates into the bulk of the metal. Strong outgassing is observed after the discharge. This behavior is reproducible from discharge to discharge.

$\mathrm{K}_{\mathrm{r}}$ can vary strongly with the actual surface composition. Stabilizing its value on stainless steel and Inconel has been achieved possible by discharge cleaning in hydrogen [19] see fig. 6. Both $D$ and $K_{r}$ are temperature dependent and the time evolution of $\mathrm{R}$ may depend also on the presence of traps for $\mathrm{H}$ atoms in the wall material. The trapping sites may be introduced by the energetic $\mathrm{H}$ particles themselves and, in future devices, by neutrons. Various models and numerical codes have been developed, describing the recycling from metals rather well [21 - 23]. An example for modeling a whole tokamak discharge in JET with Be coated walls [24] using the code PERI [22] and measured values of $K_{r}$ for $B e$ is shown in fig. 7 .

The hydride forming metal titanium, a material which has been used frequently for oxygen gettering, also getters hydrogen and thus shows a large hydrogen uptake up to the formation of the stoichiometric hydride $\mathrm{TiH}$. 
Its hydrogen pumping rate is thus transient. Phase changes introduced by the hydrogen loading and the formation of titanium oxide tend to make thick $\mathrm{Ti}$ films mechanically unstable.

The hydrogen recycling in devices of which large wall areas are covered with carbon is even more complex than that of metallic surfaces. It has been reviewed by Ehrenberg [25, 26]. Mechanistic aspects were discussed e.g. by Möller [27] and by Pillath et al. [28]. Various phenomenological models have been developed [e.g. 26, 29] involving multiple reservoirs (limiter, wall) and effective residence times or diffusion coefficients of hydrogen in the carbon.

The hydrogen recycling can vary, depending on wall temperature and prehandling, from a state in which the wall strongly absorbs plasma particles (wall pumping) to one in which the plasma is almost fully fuelled by hydrogen from the wall and in which density control is impossible [30]. Carbon absorbs hydrogen up to a saturation concentration lat room temperature) of about $0.4 \mathrm{H} / \mathrm{C}$ within the range of the impinging hydrogen particles [27]. Due to the high $\mathrm{H}$ concentration, particle induced desorption processes are important, in particular at low wall temperatures. Desorption of hydrogen by energetic He atoms from glow discharges is frequently used to provide a pumping carbon wall for a few discharges [31]. Boronization and siliconization layers (see below) can provide wall pumping for a much longer period, in particular when used on hot walls [32].

Thermal desorption is complex due to the various bonds of $\mathrm{H}$ on damaged carbon surfaces and due to the porous structure of graphites. Shown in fig. 8, as an example, is the in situ outgassing of the TEXTOR liner which had been covered with a hydrogen saturated carbon film at $200^{\circ} \mathrm{C}$ and was then ramp heated up to $400{ }^{\circ} \mathrm{C}$. The amount of hydrogen released during the $35 \mathrm{~min}$ of the ramp heating is $330 \mathrm{mbar} \ell$, about $50-60 \%$ of the hydrogen inventory of the carbon film. Subsequent tokamak discharges show less wall fueling than those performed before the bake $[30,33]$.

Codeposition of carbon atoms released from wall components and of hydrogen in deposition dominated areas of the machine provide an additional hydrogen pumping mechanism and can lead to the build up of significant hydrogen inventories.

The magnitude of the surface pumping effect depends on the area covered with the respective materials and on the fraction of the hydrogenic flux impinging on it. The locations around limiters, divertor target plates, and around the gas injection ports are probably very important in this respect because of the large local flux of charge exchange neutrals. On the other 
hand, all mechanisms discussed above lead to transient effects and saturate after a certain hydrogen fluence. High flux areas (limiters, divertor plates) saturate rapidly. It is the large wall area exposed to low fluxes, which is important for the control of recycling during longer pulses. For very long pulses or steady state operation the use of active pump limiters or pumped divertors is indispensable. Results from TEXTOR [34] and Tore Supra [35] and recently also from D IIID [36] and JET [37] with the pumped divertors indicate that the permanent particle exhaust from a pump limiter can prevent the wall from reaching the limiting hydrogen concentration. This implies that the wall pumping effect is, to some extent, existing in long pulse devices also and might allow particle control in transient phases, e.g during the current ramp down of the discharge. These aspects have been discussed in detail by Mioduszewski et al. [38].

\section{Methods of Wall Conditioning}

\subsection{Discharge Cleaning in Hydrogen and in other Reactive Gas Mixtures}

Various types of plasma discharges in a flowthrough of hydrogen gas have been used to clean in situ contaminated metal surfaces. Examples are glow discharge cleaning [39, 40], ECR-discharge cleaning [41], pulsed discharge cleaning [42, 43], Taylor discharge cleaning [44]. Several of these techniques have been reviewed by Dylla [45]. Common to all the cleaning procedures by hydrogen plasmas is the use of the mechanisms indicated above, namely the reduction of metaloxides by atomic hydrogen and also the hydrogenation of carbonaceous deposits to form volatile species in order to deplete the wall surfaces from contamination layers. The formed volatile substances (water vapor, methane and higher hydrocarbons) are desorbed from the surface both thermally and by particle impact and are evacuated from the device via the torus pumping system. It has been shown [15, 45] that it is mainly the high chemical reactivity of the atomic hydrogen species which leads to the formation of the volatile products. The cleaning rate is thus proportional to the flux density of hydrogen atoms to the wall surface. It is also important that the formed products are not reionized and dissociated in the cleaning plasma itself and redeposited on the surfaces. This requires plasmas with low $T_{e}$ and of a low ionization fraction. High wall temperatures are not only useful for increasing the rates of the reactions leading to the formation of the volatile species on the surface but also for their desorption from the surface into the gas phase. Finally, a large pumping speed for the species is required for their efficient removal out of the torus. The various methods essentially differ in the way the atomic hydrogen species are created and strike the surface. Other effects may also play a role as are sputtering due to high energy ion bombardment. 
Hydrogen glow discharge cleaning has been described comprehensively e.g. in $[39,40,46]$. One ore more anodes are either located in portholes or moved by a bellows assembly into the main volume of the vacuum vessel [33]. The wall surfaces and limiters are at ground potential. Typical hydrogen pressures, range from $5 \times 10^{-4} \mathrm{mbar}$ to $5 \times 10^{-3} \mathrm{mbar}$, the voltage between anode and cathode is of the order of $300-400 \mathrm{~V}$. It has been reported [39] that arcing occurs initially in unbaked vessels. The arcing frequency decreases quickly with prolonged glow. It is much less a problem in bakable devices. Waelbroeck et al. $[40,46]$ present a model for the temperature dependent cleaning effectiveness of a glow discharge as a function of the glow current density and hydrogen pressure with the ratio $A / S_{p}$ of the wall area $A$ and the effective pumping speed $S_{p}$ as a scaling parameter. The model describes very well the dependencies measured in a simulation vessel and in TEXTOR. Shown in fig. 9 is the removal rate of volatile impurities from TEXTOR after the initiation of a glow discharge in hydrogen. It should be noted, that the total removed amount of carbon and oxygen corresponds to a surface coverage of 100 and 200 monolayers ${ }^{*}$, respectively [47]. Similarly large quantities for the initial clean down have been measured in several devices $[39,48]$.

A comprehensive treatment of ECR discharge cleaning in toroidal devices has been given by Sakamoto [41]. So far all studies were performed at $2.45 \mathrm{GHz}$ and a toroidal field of $0.8 \mathrm{kG}$. To move the resonance zone along the major radius within the vessel, the magnetic field was usually modulated. Comparing hydrogen - ECR and glow cleaning in TEXTOR has shown about the same cleaning effectiveness for both techniques [49]. This underlines the importance of atomic hydrogen for the cleaning effect, since the dominant wall fluxes in these ECR plasmas are mostly neutral hydrogen atoms.

Whereas glow- and ECR-discharge cleaning operate continuously, pulse cleaning and Taylor-discharge cleaning use repetitive discharges whereby one of the coil systems of the tokamak is used. Taylor optimized the pulse discharge cleaning by maximizing the water vapour production [44]. This was achieved by increasing the hydrogenic filling pressure. The electron temperature fell to low enough values $(<10 \mathrm{eV})$ keeping the reionization of the released water molecules by the plasma small. The process was monitored by mass spectroscopy. Part of the cleaning action also occurs during the dwell time between pulses from implanted hydrogen atoms which are diffusing back to the surface.

In addition to mass spectroscopy, situ surface analysis techniques have been

* assuming 1 monolayer $=10^{15}$ atoms $\mathrm{cm}^{-2}$ 
used to characterize the progress in cleaning $[50,51]$. Studies on TEXTOR, ISX-A, D-III, T-12 and JET show that the final equilibrium concentration of oxygen on stainless steel and Inconel is typically between 3 at \% and 8 at \%, that of carbon is usually between 10 at \% and 30 at\% [52]. The measurements suggest the competition of different impurity species for the surface sites [52]. The removal of aliphatic and graphitic carbon proceeds rapidely. The carbon atoms at the interface to the metal have often a carbidic bond character and are much more difficult to remove.

Heavy alphatic contamination of the TEXTOR vacuum vessel had been very effectively removed by a glow discharge in He to which $5-10 \%$ of oxygen were admixed [40]. The formation of volatile $\mathrm{CO}$ occurs already at ambient temperature with a yield close to $1 \mathrm{CO}$ molecule per impinging oxygen atom. He-O glow plasmas have also been proposed for the removal of tritium containing amorphous carbon layers [33] in tokamaks and are now considered also for control of the T inventory in ITER and TPX [53]. Vice versa it has been observed, that small quantities of carbon ions in the cleaning plasma e.g. by seeding a hydrogen discharge with $1 \%$ methane [47], increases the removal efficiency for residual surface oxygen by the reciprocal mechanism.

\subsection{Glow Cleaning in Helium and Disruptive Discharge Cleaning}

Devices in which large parts of the wall are covered with carbon materials require different discharge cleaning techniques compared to those with metal walls. Usually very pure qualities of graphite are used as wall materials and wall tiles are well outgassed at high temperatures (around $1000{ }^{\circ} \mathrm{C}$ ) in vacuum before installation into a fusion device. It is the porous structure of graphite which leads to storage of significant amounts of water vapor, of the order of $1 \mathrm{mbar}$ liter/gram [54] which can be absorbed from air during the installation procedure of the tiles. When the vessel is bakable to temperatures $>300^{\circ} \mathrm{C}$, water vapor is readily desorbed. Temperatures around $150^{\circ} \mathrm{C}$ do not suffice for removal of the water vapor neither thermally nor by glow discharges [54]. In TFTR, which cannot be baked beyond $150^{\circ} \mathrm{C}$ this fact required the development of a "flash desorption" technique (termed disruptive discharge cleaning [54]) using purposefully disrupted tokamak plasmas in order to heat the carbon first wall to high enough temperatures for efficient bulk degassing.

A comparison between hydrogen and helium glow discharges has been made by Dylla [54]. It has been observed in TFTR, that similar amounts of carbon and oxygen are removed from a stainless steel and a carbon wall during an initial clean down, when hydrogen or helium glow discharges are used. This 
suggests similar reservoirs of desorbable carbon and oxygen. On stainless steel this is the oxide layer of about $20 \mathrm{~nm}$ thickness and for graphite it is the impurities within the range of the $\mathrm{H}^{+}$ions $(6-10 \mathrm{~nm})$, probably increased somewhat by $\mathrm{H}$ atom diffusion in the pores of graphite.

As mentioned above, the bulk reservoir of oxygen and of carbonaceous impurities cannot be reduced by these techniques unless performed at temperatures $>300^{\circ} \mathrm{C}$. The large tokamaks JET, JT 60-U, D III-D and Tore Supra have vacuum vessels which can be heated up to these temperatures. TEXTOR has a liner heated by direct current which can be baked up to $500^{\circ} \mathrm{C}$. It serves as a support for the graphite limiters which are then baked together with the liner. The vacuum vessel of TEXTOR is routinely baked to $300^{\circ} \mathrm{C}$.

Glow discharges in a flowthrough of helium are usually preferred over those in hydrogen for devices with large area graphite coverage. The main reason is that large quantities of hydrogen are stored within the penetration range of the hydrogen ions. They can be released upon particle impact during a tokamak discharge and make the density control difficult, in particular when the walls cannot be baked to temperatures in excess of $300^{\circ} \mathrm{C}$ (see also chapter 4). Although He does not intervene in chemical reactions, it is observed, that upon exposure to a He glow discharge, $\mathrm{CO}$ and hydrocarbon impurities are released from the carbon surfaces. As mentioned above, carbon retains implanted oxygen ions up to a concentration of about 0.25 within the range of implantation. Particle induced desorption of $\mathrm{CO}$ and of hydrocarbons by the energetic $\mathrm{He}$ ions is the primary release mechanism in helium glow discharges. An example is presented in fig. 10, showing the increase in $\mathrm{CO}$ and $\mathrm{H}_{2}$ partial pressures when a glow discharge in helium is initiated.

The desorption cross section and the depth of the affected carbon layer increase with the impact energy of the He ions. Fig. 11 shows the measured hydrogen release as a function of impact energy of $\mathrm{He}$ ions from glow plasmas and ion beam experiments on saturated carbon films and on graphite tiles from D III-D [55]. High voltages between anode and cathode, i.e. high impact energies yield the largest hydrogen desorption efficiences. Maximizing the glow voltage implies operating the helium glow discharge at low pressures. Arcing can then become a serious problem. Also included in fig. 11 is a data point for Ar bombardment [56]. Though the desorption cross section for $\operatorname{Ar}\left(3 \times 10^{-16} \mathrm{~cm}^{2}\right)$ is about one order of magnitude larger than that for $\mathrm{He}\left(4.5 \times 10^{-17} \mathrm{~cm}^{-2}\right)$ at the same impact energy of $250-350 \mathrm{eV}$, argon glow discharges are not well suited for the use in fusion devices. Argon is trapped in carbon to about $2 \times 10^{19} \mathrm{Ar}$ atoms m $\mathrm{m}^{-2}$ at $250 \mathrm{eV}$ impact 
energy and almost fully released upon bombardment with hydrogen ions of the same energy [56]. This leads to an intolerably high Ar contamination of the fusion plasma.

Helium glow discharges for hydrogen removal have been optimized in DIII-D by Jackson et al.[55]. They are routinely used at high bias voltages (i.e. high helium ion impact energy) before each tokamak discharge. They have been instrumental in achieving high performance $\mathrm{H}$ mode plasmas and a large operating space in D III-D when major parts of the surface were covered by graphite. For the initial clean down after a vent the DIII-D vessel is baked up to $350^{\circ} \mathrm{C}$. After covering all plasma facing surfaces with graphite in D III-D recently, only high temperature bake out and helium glow were used as conditioning techniques and very good plasma conditions were achieved rapidly $[58,59]$.

\subsection{Metal Film Gettering}

Metal film gettering makes use of the fact that large sticking probabilities are observed for various gaseous compounds on clean metal surfaces. Metallic tokamak wall surfaces depleted from carbon and oxygen by discharge cleaning in hydrogen show getter properties with respect to oxygen and carbon [33, 60, 61]. The controlled evaporation of metals has been used to cover large surface areas with a clean film for gettering purposes. This yields a very high conductance of the pumping area to the torus volume and thus very high effective pumping speeds. Metal evaporation is a simple technique; the equipment essentially consists of a heater surrounded by the metal to be sublimed. Commercially available systems exist for Ti since Ti-getter pumps are frequently used in UHV technology. For $\mathrm{Cr}$ - [62 - 64] and $\mathrm{Be}-$ [65] gettering special devices for the use in tokamaks were developed.

The fact that the sublimed atoms leave the hot metal surface isotropically leads to thickness patterns which are determined by geometrical effects. In the toroidal geometry of a tokamak with the constrained access to the vessel, pronounced inhomogeneities occur. Unwanted metal deposition on special surfaces like graphite limiters can be prevented by using appropriately formed baffles.

\subsubsection{Ti- and Cr-Gettering}

The sticking coefficients for molecular oxygen, nitrogen and deuterium gas on evaporated $\mathrm{Ti}$ and $\mathrm{Cr}$ films have been measured by Simpkins et al. [63]. These sticking coefficients are plotted in fig. 12 as a function of the surface 
coverage of the species on the metal film. In the case of $\mathrm{Cr}$, all sticking coefficients show a rapid decrease at a coverage of about $10^{15} \mathrm{~cm}^{-2}$, corresponding to about one monolayer. It is concluded that in the case of $\mathrm{Cr}$, the pumping of these substances is limited to the very surface layer of the metal. Pumping stops when the surface is saturated. In the case of Ti the same behavior is observed for $\mathrm{O}_{2}$ and $\mathrm{N}_{2}$ but not for deuterium.

Deuterium has a high sticking on $\mathrm{Ti}$ even when more than one equivalent monolayer is absorbed. This is due to the diffusion of hydrogen into the bulk of $\mathrm{Ti}$ and the formation of a hydride $\left(\mathrm{TiH}_{2}\right)$. The concentration of hydrogen may go up to typically $10^{23} \mathrm{~cm}^{-3}$, yielding a capacity of about $10^{4} \mathrm{mbar}$ I $\mathrm{cm}^{-3}$ for hydrogen pumping. Large fractions of the gas stored in the bulk may be released upon heating. Outgassing of Ti getter films removed from the antenna screens in Macrotor [66] show that hydrogen is released at about $380{ }^{\circ} \mathrm{C}$. It is important to note that the impurity control capacity of $\mathrm{Ti}$ and of $\mathrm{Cr}$ (removal of $\mathrm{O}_{2}, \mathrm{H}_{2} \mathrm{O}$ or $\mathrm{CO}$ ) is not reduced after the film is saturated with hydrogen. A previously adsorbed gas (hydrogen) can be replaced on the getter film by a gas with higher surface binding energy, for instance oxygen. This is confirmed by depth profiles from Auger Electron Spectroscopy (AES) on the Ti layers from Macrotor [65] which were later used for $\mathrm{H}$ desorption.

\subsubsection{Be-Gettering}

In the case of $\mathrm{Be}$, no similar data on the sticking coefficients are available. Indirect information was provided by tests which have been performed with the Be evaporators for JET in a separate test vessel. At surface temperatures of about $900{ }^{\circ} \mathrm{C}$ a reaction with $\mathrm{CO}$ and $\mathrm{CO}_{2}$ from the residual background gas occurs, leading to the formation of $\mathrm{BeO}$. The oxide impedes the further evaporation and, in addition, changes surface emissivity and the thermal insulating properties of interfaces [65]. After an opening of JET the vacuum and surface conditions are first improved by baking and glow cleaning, before Be evaporation is started (see also section 6.1). To ensure coverage of a large fraction of the wall in JET, four evaporator systems around the midplane were used during the 1991-92 campaigns [67]. It should be noted that JET usually operates with a hot wall $\left(250-300^{\circ} \mathrm{C}\right)$.

\subsection{Plasma Assisted Deposition of Thin Films (Carbonization, Boronization, Siliconization)}

For the plasma assisted chemical vapor deposition of thin films (carbonization [68], boronization [69], siliconization [9, 70, 71]) glow discharges are used which are operated in a throughflow of a carrier gas (usually $\mathrm{He}$ ) and of 
reactive gas species serving as the sources of the desired atoms (carbon, boron or silicon) from which the layers are constituted. These techniques have been pioneered by the TEXTOR team. The equipment is that normally used for glow discharge cleaning: one or more anodes in the tokamak vessel, a continuous gas feed and the torus pumping system. When hazardous gases $\left(\mathrm{B}_{2} \mathrm{H}_{6}, \mathrm{SiH}_{4}, \mathrm{~B}_{10} \mathrm{H}_{14} \mathrm{~B}\left(\mathrm{CH}_{3}\right)_{3}\right)$ or their deuterated equivalents are used, special components and operation procedures of gas injection and pumping systems may be required to ensure safe handling [72].

The molecules of the reactive gases are partly ionized in the glow plasma and accelerated in the cathode sheath onto wall and limiters or divertor plates which are the discharge cathode (see fig. 13). The growth processes may be complex involving neutral radicals and positive and negative molecular ions. The stoichiometry of the deposits can be controlled by the composition of the reactive gas species. Carbon films are deposited usually from $\mathrm{CD}_{4}$ (carbonization), $\mathrm{B}$-layers from $\mathrm{B}_{2} \mathrm{D}_{6}$, for example (boronization). For the deposition of silicon layers (siliconization) deuterated silane $\mathrm{SiD}_{4}$ and disilane $\mathrm{Si}_{2} \mathrm{D}_{6}$ have been used $[70,71]$. The films applied to fusion devices have mean thicknesses of about $50 \mathrm{~nm}$ to $100 \mathrm{~nm}$.

Different precursors have been used for boronization by glow discharges: diborane $\mathrm{B}_{2} \mathrm{D}_{6}$ [69], trimethylboron $\mathrm{B}\left(\mathrm{CD}_{3}\right)_{3}$ [73-75], decaborane $\mathrm{B}_{10} \mathrm{H}_{14}$ [76], and carboranes $\mathrm{C}_{2} \mathrm{~B}_{10} \mathrm{H}_{12}$ [77]. The latter two substances are solids and have to be heated in excess of $100^{\circ} \mathrm{C}$ to have a high enough vapor pressure for seeding a helium glow discharge. Carboranes are claimed to be less hazardous than the other compounds. A recent summary of boronizing russian tokamaks by carboranes was presented by Sharapov et al. [78]. Tabarés et al. compare boronization by trimethylboron and decaborane in the small tokamak TJ-1 [79]. A comparison of glow discharge boronization, boronization by gas injection into a tokamak plasma (both using trimethylboron), and of "solid target" boronization was presented by Boucher et al. [75].

The coverage of the surfaces is usually not uniform. It has been observed during the initial boronizations in TEXTOR and in D III-D that the layer thickness decreases in toroidal direction from the point of gas introduction with a characteristic decay length of about $1 \mathrm{~m}[80,81]$. This is due to the rapid local ionization, fast deposition and the resulting depletion of diborane in the gas mixture of the glow discharge. Using high flow rates, toroidally distributed multiple gas injectors, and "pulsed glow" [81] have improved the homogeneity.

The carbonization process produces amorphous hydrogen rich "diamond-like" carbon films a-C:H [33, 82]. About $60-65 \%$ of the carbon atoms are sp-3 coordinated (diamond), about $25-30 \%$ are in the graphitic coordination and 
a fraction of about $10 \%$ is in aliphatic bonds. The $\mathrm{H} / \mathrm{C}$ ratio is 0.4 when the deposition occurs at $\mathrm{T} \leq 150^{\circ} \mathrm{C}$. The film is transparent, hard, and homogeneous down to a microscopic scale. Experiments using the annihilation of positrons revealed, that at least $3 \%$ of the total volume is occupied by micropores with a diameter of about $0.5 \mathrm{~nm}$ [83, 84]. Auger Electron Spectroscopy with sputter depth profiling (AES) only detects carbon. No other impurities are found in the bulk. The film is inert to air exposure. Carbonization provides an all carbon coverage of the entire inner vessel surface. The hydrogen recycling, thermal hydrogen desorption and the effects of a helium glow on a carbonized surface are identical to that of the surface layer of hydrogen implanted graphite. This can be expected from the almost identical surface structure and composition of both materials [85].

Boronization produces amorphous boron containing carbon films a-C/B:H [69, 86]. The prime advantage of the boronization films is their ability to getter oxygen due to the incorporated boron atoms and their superior resistance to chemical erosion by thermal and energetic hydrogen species [11, 87-89]. Best $O$ gettering properties are found for layers rich in B (pure B films) whereas lowest erosion rates by hydrogen found for the stochiometric composition of boron carbide $\mathrm{B} / \mathrm{C}=4$ [87]. Depending on temperature the hydrogen erosion yields for films with a ratio $B / C-1$ are lower by a factor up to $10-20$ compared to $\mathrm{a}-\mathrm{C}: \mathrm{H}$ or graphite irradiated by energetic hydrogen ions [88]. Radiation enhanced sublimation is not significantly suppressed in $\mathrm{a}-\mathrm{C} / \mathrm{B}: \mathrm{H}$. Boronization layers have a well defined composition which is homogeneous in depth. No noticable oxygen contamination of the bulk film occurs, even after storage in air for several weeks. X-ray induced Photoelectron Spectroscopy (XPS) reveals that the $\mathrm{B}-\mathrm{C}$ bonds in a-C/B:H have a pronounced carbidic character [86]. This is probably the reason for the chemical inertness and the improved thermal stability compared to a-C:H $[86,89]$. Thermal desorption, nuclear reaction, and ion beam analysis yield a concentration of $\mathrm{H} /(\mathrm{B}+\mathrm{C})=0.3-0.4$ for films prepared at ambient or elevated temperatures up to $300^{\circ} \mathrm{C}$. This is close to the value for $\mathrm{H} / \mathrm{C}$ in a$\mathrm{C}: \mathrm{H}$. Detailed measurements on the dependence of the $\mathrm{H}$-concentration on the precursor gases $\mathrm{B}_{2} \mathrm{H}_{6}$ and $\mathrm{B}_{10} \mathrm{H}_{14}$ and on the deposition temperature were described by Yamage [90]. Experiments with slow positron beams [91] indicate a considerable microporosity with a mean pore size of about $1 \mathrm{~nm}$ diameter, which is larger than in a-C:H. In contrast to pure carbon surfaces, oxygen gettering occurs when the boron containing surfaces are exposed to energetic $\mathrm{O}$ particles. XPS clearly reveals the formation of $\mathrm{B}_{2} \mathrm{O}_{3}$ [92] which is a solid with low vapor pressure. When the bombardment is stopped, $B / C$ surfaces appear to be chemically stable.

Adsorbed (molecular) diborane gas on tokamak wall surfaces can also act as a getter for oxygen. The effect has been discussed in detail in [93]. It is 
assumed that $\mathrm{B}_{2} \mathrm{H}_{6}$ reacts preferentially with residual metal oxides on untreated surfaces of the vacuum system according to the following schematic reaction:

$$
\mathrm{B}_{2} \mathrm{H}_{6}+3 \mathrm{MO}->\mathrm{B}_{2} \mathrm{O}_{3}+3 \mathrm{M}+3 \mathrm{H}_{2} .
$$

This reduces the release of water vapor in reactions with hydrogen and easily reducible metal oxides $\left(\mathrm{NiO}, \mathrm{Fe}_{2} \mathrm{O}_{3}\right.$ etc) and thus decreases the primary oxygen source in the tokamak. A reduced outgassing of $\mathrm{H}_{2} \mathrm{O}$ is measured after this procedure and improved plasma performance is observed [93].

Siliconization results in amorphous silicon containing carbon films a-Si:D, a-Si/C:D, or a-Si/B/C:D, depending on the gas mixture used during the film deposition. The stochiometry can be well controlled within the full Si-C-B ranges. The film properties are described in detail in [71]. The layers are semitransparent with a refractive index $n=(2 \pm 0.2)$ and have a low absorption coefficient $k<0.01$. The density $\rho$ of films incorporating carbon is $\rho=(1.5 \pm 0.2) \mathrm{gcm}^{-3}$. Carbon free coatings have $\rho=(2 \pm 0.1) \mathrm{gcm}^{-3}$. The ratio of hydrogen to silicon atoms in carbon free layers is $\mathrm{H} / \mathrm{Si}=0.2$, whereas the addition of carbon and boron increases the hydrogen content. a-C/Si:H produced in purely rf excited plasmas shows a pronounced peaking of the hydrogen concentration at a $\mathrm{Si} / \mathrm{C}$ ratio of about 0.4 , reaching a ratio of $\mathrm{H} /(\mathrm{Si}+\mathrm{C})$ close to 1 [94]. The binding state of silicon atoms in a-C/Si:D is of the SiC carbidic bond type as measured by XPS [71]. It can thus be expected that the films are resisting chemical erosion. Mass spectroscopy of the residual gas during chemical erosion of a-C/Si: $\mathrm{H}$ with $\mathrm{Si} / \mathrm{C} \sim 1$ by a hydrogen plasma at $250^{\circ} \mathrm{C}$ yields $\mathrm{CH}_{4}$ release rates of $1.5 \times 10^{-3} \mathrm{CH}_{4} / \mathrm{H}^{+}$ which are about a factor of $\mathbf{3 0}$ lower than in carbonization films and a factor of 2 - 3 lower than that of $\mathrm{a}-\mathrm{C} / \mathrm{B}: \mathrm{H}(\mathrm{B} / \mathrm{C} \sim 1: 1)$ at the same temperature [71]. The methane formation is transient. Once the carbon atoms are removed within the range of the impinging hydrogen ions, the hydrocarbon formation decreases to virtually zero. No volatile $\mathrm{Si}-\mathrm{H}$ products were found in the residual gas. They may have a large sticking coefficient on the wall and thus not show up in these measurements. When silicon layers are bombarded with oxygen, high sticking coefficients close to unity are observed. Whereas a transient $\mathrm{CO}$ production is observed in a-C/Si: $\mathrm{H}$ films, removing all available carbon within the range of the impinging $\mathrm{O}^{+}$ions, pure Si films show gettering of the oxygen ions from the very beginning. The effective sticking coefficient of $\mathrm{O}^{+}$ions as a function of the oxygen ion dose is shown in fig. 14 and agrees well for the pure Si film and for a-C/Si:H, corrected for CO production. A getter capacity for oxygen of $1.5-2 \times 10^{22} \mathrm{O}$ atoms is deduced from these data for the siliconized TEXTOR with a plasma exposed surface area of about $35 \mathrm{~m}^{2}$. 
Boronization has been applied at various wall temperatures $T_{W}$ (TEXTOR: $100^{\circ} \mathrm{C}-350^{\circ} \mathrm{C}$, DIII-D > $250^{\circ} \mathrm{C}, \mathrm{JT}-60 \mathrm{U}>250^{\circ} \mathrm{C}$, most other devices at room temperature). The most pronounced effect is on the hydrogen concentration which decreases with increasing temperature. Thus also the hydrogen recycling is lower when the films are deposited hot.

It has also been shown, that boronization films grown at high temperature tend to absorb less water vapor when stored in air for a long time [87], indicating that the microstructure might also be affected by the deposition temperature.

In the case of carbonization, the graphitic fraction of carbon atoms increases above $250{ }^{\circ} \mathrm{C}$ at the expense of the diamond like sp-3 coordinated carbon atom fraction [95]. For fusion applications, the effect of $T_{W}$ on the hydrogen concentration and on recycling is the dominant one in this case also.

Siliconization experiments have been performed in TEXTOR at $2500^{\circ} \mathrm{C}$ and in FT-U at $25^{\circ} \mathrm{C}$ [96]. The database is still too small to identify trends of the effects of $T_{W}$.

\subsection{Film Deposition during Tokamak Discharges}

Trimethylboron [75, 97-99], diborane [100] and silane [70] have been injected into the scrape-off-layer (SOL) during tokamak discharges. The boron, carbon and silicon atoms have a high sticking coefficient on the wall, in contrast to the hydrogenic atoms of the parent molecule. The latter fuel the plasma with the same efficiency as deuterium gas $[98,99]$ whereas the low $Z$ impurities are well shielded by the SOL from penetrating into hot plasma zones. The radiated power fraction and $Z_{\text {eff }}$ are essentially unchanged compared to $D_{2}$ fueled discharges and the performance of the tokamak plasmas is not adversely affected. When reactive gases are injected at very high rates, a cooling of the plasma boundary below about $10 \mathrm{eV}$ may occur leading to a sudden loss of the shielding property of the SOL [70]. The critical injection rate increases with heating power.

Solid boron containing materials $\left(\mathrm{B}_{4} \mathrm{C}\right.$ and $\left.\mathrm{B} / \mathrm{C}\right)$ were heated by plasma exposure within the SOL up to temperatures at which boron selectively sublimated and thereby seeded the SOL [75, 101-103]. The sublimation rate is difficult to control, however. Experiments in TEXTOR during which the surfaces of $\mathrm{B}_{4} \mathrm{C}$ limiters were heated beyond the melting point did not show a significant effect on the plasma core properties in spite of the large sublimation rates of boron. A noticeable dilution of hydrogen in the SOL and an increase of sputter erosion on other wall elements occurred, however [101]. 
Pellets, as in the case of Li-pellet injection in TFTR [104], penetrate the last closed flux surface (LCFS). The ablated material will diffuse radially outward and may coat preferentially the areas in contact with the LCFS.

All the coating techniques using the tokamak plasma itself lead to inhomogeneous layer deposition because the ionized species are guided by the magnetic field. Though still in the phase of development, the potential advantage of these techniques is their application realtime during the discharge, making them particularly suited for steady state devices.

\section{Influence of Wall Conditioning on Tokamak Discharges}

In this chapter the wall conditioning techniques, as described in the preceding paragraphs, are discussed with respect to their effects on plasma impurity content, hydrogen recycling and plasma performance.

\subsection{Discharge Cleaning in Hydrogen and Helium}

Conventional pulsed discharge cleaning, i.e. low grade tokamak plasmas, has been used prior to 1975 and is reported to be rather inefficient for the clean down of the vacuum vessel [45]. About $10^{4}$ or more of these pulses were needed to lower the carbon plasma impurity concentration $n_{c} / n_{e}$ to about $1 \%$, whereas it has been very difficult to reduce the oxygen contamination below about $5 \%$. A side effect of the repetitive tokamak plasma pulses has been a gradual heating of the wall due to eddy currents in the vessel structure. This has been advantageous when the vacuum vessel could not be baked by external means. Taylor-type discharge cleaning has been studied in several devices, e.g. in TFR [105], PLT [106], ISX [107] and JFT-2 [108] and has often been combined with Ti gettering (see below).

Most of the large tokamaks use glow discharges as part of the cleaning recipe. Studies on the first clean up of the vacuum system or after a major machine opening have been published e.g. from ASDEX [109], PDX [39], TFTR [110, 111], TEXTOR [47], JET [112, 113], Doublett III [114]. An example for the release of volatile impurities has been shown in Fig. 9, already.

Glow- and ECR-discharge cleaning in TEXTOR at wall temperatures of $150^{\circ} \mathrm{C}$ and at $300^{\circ} \mathrm{C}$ are compared with respect to the achievable values of $Z_{\text {eff }}$ in subsequent tokamak discharges [115]. The data are summarized in fig. 15. The figure also contains a data point refering to a hydrogen glow discharge 
seeded with methane to enhance the oxygen removal. TEXTOR has been operated with all metal walls and metal limiters routinely at $Z_{\text {eff }} \approx-3$ for medium densities of about $3 \times 10^{13} \mathrm{~cm}^{-3}$. The ohmic density limit has been around $3.5 \times 10^{13} \mathrm{~cm}^{-3} . Z_{\text {eff }}$ and the brilliance of metal lines increase as the density is lowered in ohmic discharges (while $T_{e}$ increases accordingly). This is attributed to sputter erosion of metals by low $\mathrm{Z}$ impurities, mostly oxygen. Similar to the experience in TFR problems due to metal liberation occurred when ICRH heating was applied. Excessive radiation prevented the coupling of more than $200-300 \mathrm{~kW}$. The conditions were improved substantially after carbonization of TEXTOR.

Another study of the correlation between wall conditioning and tokamak plasma performance has been made on TFTR [54, 110] using ex situ bake out of the movable graphite limiter and both hydrogen glow discharge and pulse discharge cleaning. Values of $Z_{\text {eff }}=2-3$ have been obtained at the observed density limits $\left(n_{e}<3.5 \times 10^{13} \mathrm{~cm}^{-3}\right)$ for plasma currents of 1.4 MA and a toroidal field of $2.7 \mathrm{~T}$.

JET reports on the effects of their cleaning procedure for the first operation of the device which had an Inconel wall and four discrete graphite limiters [112]. The cleaning involved mainly baking and hydrogen glow cleaning. Long ohmic pulses of ten seconds were rapidely achieved but $Z_{\text {eff }}$ was between $3-5$ with about $70-80 \%$ of the radiation coming from nickel and other medium $Z$ elements, see fig. 16.

Large areas of the walls of the mid-size and big tokamaks are now covered by graphite or carbon fibre composits (CFC's). Again high temperature bake out and glow cleaning in hydrogen and helium is an important part of the cleaning procedure (see sections 5.1 and 5.2). As already discussed above, helium glow discharge desorption of hydrogen and of $\mathrm{CO}$ stored in the surface layers of graphite has been found to very effective for obtaining reproducible high performance plasmas. As discussed by Jackson [55] reducing the deuterium gas fueling by preceding He glow has led to an expansion of the operating space of D III-D. The H-mode power threshold, which scales in D III-D empirically like

$$
\mathrm{P}_{\mathrm{L}>\mathrm{H}} \sim \mathrm{n}_{\mathrm{e}} \cdot \mathrm{B}_{\mathrm{T}} ;
$$

was lowered by reducing the density of the ohmic target plasmas. ELM-free phases are prolonged due to the less intense wall fueling during $\mathrm{H}$-mode. New regimes in D III-D (ohmic $\mathrm{H}$-mode, limiter $\mathrm{H}$-mode) were only discovered after intense He glow. More recently, after full coverage of DIII-D with graphite it has been possible by baking and $\mathrm{He}$ glow alone to achieve $\mathrm{VH}$-mode(very high confinement) plasmas of good quality. $\mathrm{VH}$ mode was 
found previously only after boronization of the device [116], see below.

\subsection{Medium Z Gettering ( $\mathrm{Ti}$ and $\mathrm{Cr}$ )}

A detailed survey of the effects of $\mathrm{Ti}$ - and $\mathrm{Cr}$-gettering on plasma performance was presented in a previous paper [93]. All large tokamaks use presently low $Z$ oxygen gettering schemes (boronization, siliconization, beryllium evaporation); $\mathrm{Ti}$ - and $\mathrm{Cr}$ - gettering are no longer widely applied. Historically they have played an important role and the mechanisms identified in the early works are still valid. Thus some of the key findings are summarized here.

\subsubsection{Ti-gettering}

A pioneering paper on Ti gettering in the ATC tokamak was published in 1975 by Stott, Daughney and Ellis [117]. Ti was initially evaporated in one half of the vessel covering about $25 \%$ of the torus surface and the stainless steel limiters. The base pressure of the machine was reduced from 2-3 $x$ $10^{-7}$ Torr to $4 \times 10^{-8}$ Torr, the plasma resistance dropped by $15-30 \%$ and $Z_{\text {eff }}$ was reduced from 5-9 before to $1.4-1.2$ after gettering. The density control achieved by the hydrogen pumping of $\mathrm{Ti}$ and the reduced impurity influx required a factor of 3 more gas to achieve the same density as before and allowed a gas puff during the discharge, cooling the plasma periphery. Coating the other part of the torus without the limiter had the same result.

This early work on ATC was soon followed up in DITE [118], confirming and further quantifying the findings in ATC, in particular with respect to the achievable density limit. Without gettering the oxygen influx increased in parallel with gas puffing and density build up. Disruptions at the density limit were correlated with excess radiation, in particular from 0 . After gettering the $O$ influx was strongly reduced and the density limit enhanced. Gettering soon preceded every discharge. $Z_{\text {eff }}$ of 1.2 was achieved in high density discharges with central values of $n_{e} \sim 6-7 \times 10^{13} \mathrm{~cm}^{-3}$.

ISX-A [119] reported in 1978 about difficulties in isotope control after heavy $\mathrm{Ti}$ gettering due to the large hold up of hydrogenic species, making the change over from $H$ to $D$ very slow.

Ti- gettering in PLT [120]) led to almost a doubling of the density limit as compared to the discharge cleaned torus. The oxygen concentration was typically reduced by a factor of 3 and remained low for a series of discharges unlike in the discharge cleaned device where it increased from shot to shot. 
Strong additional heating by beam injection which became possible at low densities of $n_{e}=1 \times 10^{13} \mathrm{~cm}^{-3}$ yielded very high ion temperatures.

Heavy Ti gettering was used in the divertor areas of PDX [121] to achieve high pumping speeds for hydrogen. The area was about $5 \%$ of the total inner surface. $Z_{\text {eff }}$ values close to 1 were achieved. It was reported that flaking of thick Ti layers occurred, leading to strong disturbance of plasma operation.

It was found in ISX-B [122] that it is sufficient for impurity control to have one or two getter cycles per operation day, whereas the hydrogen pumping deteriorates after 10 discharges. The metal contamination ( $\mathrm{Ti}$ and $\mathrm{Fe}$ ) is decreased in ohmic discharges but not for beam heated plasmas. The metal release in ISX-B is dominated by charge exchange sputtering from the walls.

The beneficial effect of Ti- gettering are the very efficient control of the oxygen impurity influx. The deposition of the getter material on the limiters is not important. It is the large wall surface where Ti reduces the recycling of both oxygen and carbon impurities. Metal release is reduced at low heating powers, probably due to the elimination of 0 sputtering. The improved plasma purity and the hydrogen pumping action of Ti allows strong additional gas puffing which cools the plasma periphery and thereby further reduces the impurity release. $\mathrm{Ti}$ getter experiments did not allow to unambiguously discriminate between the relative importance of the two effects. Critical aspects of Ti- gettering are the huge storage of hydrogen isotopes and the embrittlement of the films leading to flaking. The first point is particularly cumbersome for devices which use tritium and was the main motivation to develop the getter scheme using $\mathrm{Cr}$, which should also control low $\mathrm{Z}$ impurities but not pump hydrogen, see fig 12 .

\subsubsection{Cr-Gettering}

$\mathrm{Cr}$-gettering was first performed in Macrotor [66] in 1980 and compared to that of $\mathrm{Ti}$. It was found that the control of oxygen impurities was equally good for both schemes. As expected the hydrogen recycling on $\mathrm{Cr}$ is much larger than that on $\mathrm{Ti}$.

$\mathrm{Cr}$ gettering was also applied to ISX-B [123] where the impurity control was similarly effective as with Ti gettering. The fact that no hydrogen pumping occurred with the impurity gettering meant the impurity control was responsible for the observed plasma performance in gettered tokamaks.

The largest tokamak which used $\mathrm{Cr}$-gettering was TFTR [124]. Covering $70 \%$ of the torus by $\mathrm{Cr}$ led to a decrease of a factor 2 in oxygen impurity 
radiation, an increase in the plasma density limit by $20 \%$ and to a decrease of $Z_{\text {eff }}$ near the density limit from 1.4 to 1.1 . Care was taken not to-cover the graphite limiters with $\mathrm{Cr}$.

No storage of hydrogen by $\mathrm{Cr}$ occurs; its hydrogen recycling properties are adequately described by the dynamic "wall pumping", see e.g. [19]. Large inventories as caused by the high solubility of hydrogen in Ti do not occur, see chapter 5.3.1. The studies on $\mathrm{Cr}$ - and $\mathrm{Ti}$ - gettering have shown that strongly heated plasmas tend to liberate the metals from the wall, in particular when operated at low density. This causes problems due to their rather high value of $Z$.

\subsection{Plasma Assisted Thin Film Deposition and Be Evaporation}

\subsubsection{Carbonization}

Carbonization, leading to an "all carbon" inner surface coating, was first applied late 1984 in TEXTOR and has since been used in many other devices like ASDEX [125], JET [126], JIPP T II-U [127], D III-D [128], Heliotron - E [129], etc. The effects have been discussed in a comprehensive manner in [33].

The general observation is the almost complete suppression of metal impurity atoms in the plasma since the entire wall surface is covered with the clean carbonaceous film. This has led to a breakthrough in heating fusion plasmas by electromagnetic waves [130] and to a general improvement in plasma performance. This became manifest by low values of $Z_{\text {eff }}$ and a low fraction of $\mathrm{P}_{\text {rad }} / \mathrm{P}_{\text {heat }}=0.2-0.4$ of total radiated power $\mathrm{P}_{\text {rad }}$ to that of the total heating power $P_{\text {heat }}$.

Oxygen as a plasma impurity is reduced by carbonization (factor of about 5 - 8) compared to the well discharge cleaned metallic surfaces. This strong reduction is transient (over about 20 discharges) and a gradual increase of oxygen, saturating at about a factor of 2 - 3 net reduction is observed. The reason is the complex cycle of oxygen in an all carbon machine, already discussed in chapter 4. 


\subsubsection{Boronization, Siliconization and Be Gettering}

\subsubsection{Influence on Plasma Impurities and Density Limits}

A detailed comparison of boronization and the effects of Be gettering in JET has been presented in [93]. The first paper on the Boronization of tokamak walls has been published from TEXTOR in 1989 [69]. In the meanwhile the technique has been adopted by many devices and has become a preferred conditioning method in tokamaks, stellarators and reversed field pinches [ASDEX [131], TCA [132], TFTR [133], D III-D [134], JT-60 U [76], Tore Supra [135], W7-AS [136], Repute-1 [137], and other machines].

Three main effects are observed following the application of these low $Z$ wall coatings: suppression of (high Z) metal liberation from the vessel wall, reduction of the low- $Z$ impurity concentrations in the plasma, in particular of oxygen, and a reduced hydrogen recycling [32].

The metal release from the first wall is suppressed because of the coverage of the structural material and its protection by the coating. The wall is usually well shielded from direct plasma impact by the high heat flux components and it is mostly the flux of charge exchange neutrals which hits its surface. These fluxes are low and thus the lifetime of the coatings on the wall is high, of the order of many weeks of operation in present devices (see chapter 6.3.3).

The reduction of the low- $Z$ impurities involves more subtle mechanisms than in the case of surface gettering by $\mathrm{Ti}$ and $\mathrm{Cr}$. In the case of boronization it has been shown by surface analysis of layers deposited on collector probes in the SOL of TEXTOR, that oxygen is retained in these deposited layers as a non volatile oxide [138]. Figure 17 shows the B1s photoelectron line of the boron atoms in this redeposited layer as measured by $X$-ray induced Photoelectron Spectroscopy. Some of the B atoms show a carbidic bond structure (shoulder at $189 \mathrm{eV}$ ) as have all atoms in the virgin boronization film (reference). The largest fraction of $B$ atoms however shares bond electrons with $\mathrm{O}$ atoms, as indicated by the peak around $192 \mathrm{eV}$, which confirms the formation of boronoxides. The reciprocal relation between the oxygen plasma impurity concentration and the amount of oxygen gettered in the redeposited film has been documented in TEXTOR by a series of collector probe measurements at various times following a boronization [138]. Similar observations have been made for redeposited layers after beryllium gettering in JET [139]. The control of oxygen impurities occurs via gettering by the active atoms $\mathrm{B}, \mathrm{Be}$, or Si not only on the surface of the deposited wall coating but also within redeposited layers in deposition dominated areas of the machine. The high heat flux areas on divertor plates 
and limiters do not contribute, since they are erosion dominated.

The reduction of the low $\mathrm{Z}$ impurity level after boronization, siliconization and beryllium evaporation is seen clearly when e.g. the line emissions of oxygen at the limiters, e.g. Ol, OII and CII, normalized to the deuteron fluxes are compared to those found in a carbonized vessel. As discussed in detail in [93], the reduction is a factor of 3 in TEXTOR for both $O$ and $C$, and can be significantly higher (factors of 10 or larger) depending to which state of previous machine condition the comparison is made. The decrease of the $\mathrm{C}$ impurity level is attributed mostly to the suppression of $\mathrm{CO}$ formation. This has been confirmed by sniffer probe measurements in the SOL [69].

In comparing the oxygen absolute fluxes between boronization and siliconization, a further reduction by a factor of up to two is observed, see fig. 18. This is attributed to the even higher oxygen affinity of $\mathrm{Si}$ as compared to $\mathrm{B}$. A decrease of the $\mathrm{C}$ impurity level in parallel with that of oxygen is also observed after siliconization.

The reduction of oxygen in JET after Be evaporation is at least a factor of 10. The flux ratio of carbon atoms and deuterons at the limiter is reduced to about $3 \times 10^{-2}$ at $n_{e}>1.5 \times 10^{13} \mathrm{~cm}^{-3}$ for ohmic plasmas and has about the same value as in TEXTOR after boronization. The equilibrium level of $C$ in devices with high heat flux components from graphite is finally determined by their sputtering due to plasma deuterons and due to selfsputtering.

The efficient impurity control by the low $Z$ gettering films has made it possible to achieve values of $Z_{\text {eff }}$ below 2 at $n_{e}$ as low as $1 \times 10^{13} \mathrm{~cm}^{-3}$, and approaching 1 at higher densities [93]. Such ohmic target plasmas are essential for achieving e.g. the hot ion mode, I-mode or supershot high confinement modes with additional heating.

Other striking effects of thin films are the reduction of radiated power after boronization and Be evaporation. The radiated power fraction shows only a slight increase with density. At the density limit the global radiation from the bulk plasma is only about $30 \%$ of the input power. In these cases often MARFES (poloidally localized highly radiative cold plasma zones) occur leading to a local radiative collapse. The radiation properties of a siliconized machine, e.g. TEXTOR, are different and shown in fig. 19. At low densities sputter erosion and the high radiation potential of Si lead to a large radiated power fraction, which decreases to low levels at high $n_{e}$ when sputtering vanished and the shielding of the SOL for $\mathrm{Si}$ increases. All radiation occurs from a shell in the plasma periphery. This makes siliconization interesting for studies with a radiative plasma boundary and for very high density operation. 
Easy recovery from disruptions has been observed in JET after beryllium evaporation and in all devices using boronization [93]. It is attributed to the control of oxygen impurities.

Wall coatings have led to increased density limits. It is reported from JET [66] that the maximum density depends on the total heating power as $P_{\text {tot }} 0.5$ after beryllium evaporation and that the limit is "soft" in the sense that repetitive MARFES (poloidally localized highly radiative cold plasma zones) can form without inducing a major plasma disruption. The same phenomena are observed on TEXTOR for both boronization and siliconization $[93,32]$. It is particularly noteworthy that the maximum attainable density in the siliconized TEXTOR is about $30 \%$ higher than that after a boronization (under similar operation conditions) although the atomic number of $\mathrm{Si}$ is almost a factor of 3 higher than that of B. It may be speculated that the dominant impurity species and thus the radiation proceses of the MARFE are significantly different with different wall coatings. This may lead to a different appearance of the density limit.

\subsubsection{Influence on Hydrogen Recycling}

The hydrogen recycling during tokamak discharges can be described in terms of the wall pumping rate, which is defined as

$$
\Phi_{\mathrm{W}}=\Phi-\mathrm{dN}_{\mathrm{tot}} / \mathrm{dt}
$$

where $\Phi$ is the rate of external gas injection, including Neutral Beam Injection, and $\mathrm{N}_{\text {tot }}$ is the total number of hydrogenic atoms in the discharge. $N_{\text {tot }}$ equals the total number of electrons, corrected for contributions from impurities.

Shown in fig. 20 is the wall pumping rate $\Phi_{\mathrm{w}}$ for two discharges in JET after beryllium evaporation and for an all carbon environment [140]. The strong dynamic pumping, expected (see section 4) for the non hydride forming metal $\mathrm{Be}$ is evident. It should be recalled here, that JET has obtained its best performance in $\mathrm{VH}$ mode plasmas under these conditions.

Boronization and siliconization layers behave differently than Be and $\mathrm{C}$. An example is given in fig.21 for TEXTOR after a siliconization, showing $\Phi_{w}$ as a function of time during a discharge, the respective time trace of $\mathrm{N}_{\text {tot }}$, and the dependence of $\Phi_{w}$ on $N_{\text {tot }}$ for two consecutive series of discharges. Strong wall pumping is observed. The pumping rate does not continue to decrease during the discharge as in the case of beryllium but saturates at a level of about $1 \times 10^{21} \mathrm{~s}^{-1}$. The rapid decrease of the signal at $t=2 \mathrm{~s}$ in fig. 
5 is due to the termination of the gas feed. $\Phi_{w}$ increases as $N_{\text {tot }}$ is raised, but shows a gradual decrease as function of discharge number, which is about a factor of 2 after 20 discharges. The same qualitative behaviour is observed for a boronized machine, with wall pumping rates which are typically a factor 5-10 smaller than in the case of siliconization, however. Still this amount of wall pumping and the capacity exceed those of a carbon wall even when pretreated by He glow discharges. The reasons are not yet fully understood. Recent laboratory data suggest that hydrogen is released at much lower temperatures from $B$ - bonds than from $C$ bonds [141]. There is little evidence for a significant "dynamic" pumping effect. It has been measured, however, that pure B and Si films store less hydrogen than layers which incorporate carbon in addition. The process of forming mixed layers, increasingly enriched in carbon, occurs during erosion and redeposition in a boronized or siliconized tokamak with graphite high heat flux components. The growing redeposited layers thus can store additional hydrogen, which may contribute to the large rates of wall pumping.

Analysis of $\mathrm{VH}$-mode discharges in D III-D suggests that the enhanced wall pumping of helium glow treated boronized surfaces as compared to carbon surfaces is instrumental for obtaining the observed very high performance discharges in D III-D [113, 134], see fig. 22. Similar observation have been made on JT-60 U in very high confinement plasmas in a combined high $B_{\text {pol }}$ / $\mathrm{H}$-mode where low recycling associated with high confinement was obtained after boronization [142], fig. 23.

\subsubsection{Lifetime of Coatings}

The lifetime of a coating is measured by the length of plasma exposure during which it has an influence on the plasma properties. Depending which quantities are considered, the lifetime may be significantly different, e.g. for recycling control, oxygen impurity control or for providing a radiative plasma boundary. An example is shown in fig. 24 for a siliconization in TEXTOR [69]. Following the siliconization, the lowest carbon and oxygen impurity levels ever observed on TEXTOR were measured during about 50 discharges. Thereafter the $\mathrm{O}$ and $\mathrm{C}$ levels increase to values of typically $2 \%$, characteristic for an "old" siliconization and boronization in TEXTOR. The silicon fluxes decrease by about a factor of 2 during this period but are still high enough to provide a strongly radiative plasma boundary. The lifetime of a few $100 \mathrm{~nm}$ thick coating on the high heat flux components depends strongly on the local plasma parameters but is generally limited to a few 100 s net plasma exposure. Shown in fig. 25 are the decrease of the Bll and of the Bel line radiations in front of the toroidal limiter in TEXTOR and in front of the belt limiter in JET, respectively. TEXTOR had been freshly boronized 
and JET had been coated with beryllium just prior to these measurements. Though the intensities decrease rapidly as a function of the discharge number, indicating the local erosion of the coating, a low but constant boron, respectively beryllium level is maintained. The latter are due to a erosion/deposition equilibrium which changes only slowly. The effect of the wall coating wears off less rapidly due to redeposited material with is increasingly enriched in carbon from erosion of the graphite high heat flux components. The coatings thus have to be replenished from time to time, typically every 3-5 weeks of operation in TEXTOR. Fuelling of reactive gases (silane) in the ramp-down phase of the discharge has been shown to be effective in prolonging the lifetime of the coating significantly [143]. When bulk beryllium is used as divertor target plate or limiter material in JET and refreshes the surface layer by evaporation the lifetime of Be coatings may be significantly increased. This beneficial effect has to be weighed against high erosion rates, which will probably be unacceptable in steady state devices.

\subsection{Li-pellet injection}

TFTR found empirically that the injection of Li pellets leads to conditioning of the graphite limiter surfaces and increases significantly the performance of the plasmas [104]. Li pellet injection was instrumental in achieving record values of $n_{i} T_{i} T_{e}$ and of the fusion power generation in D-T discharges. Best performance and highest energy confinement is obtained with the injection of several Li-pellets before the beam heating phase. The effects are shortlived and last only one discharge. It has been observed in TFTR that the recycling hydrogenic flux, as measured by $\mathrm{D}_{\sigma}$-emission, and the $\mathrm{Cl}$ line radiation decrease with increasing amount of $\mathrm{Li}$ and that their decrease correlates with the increase in energy confinement. This correlation is not surprising, however, since energy and particle confinement are usually linked and better particle confinement leads to reduced wall fluxes of deuterons and thus to a decrease in impurity generation. The mechanisms by which $\mathrm{Li}$ is effective have not been identified yet. Injection of boron or carbon pellets has been less successful [144].

Recently, Li-pellet injection was implemented on DIII-D [145] which has a full graphite coverage of its plasma exposed surfaces. The pellets were fired at the end of a beam heated discharge and ablated at a normalized minor radius $\rho \sim 0.7$. On the next discharge after Li-pellet injection Lil emission from the divertor region was observed whereas no Li l light was measured around the main chamber. This indicates that the Li l-ions are guided along the magnetic field and deposited at the divertor target plates. This effect only lasts one discharge. However, accumulative reduction of the (already low) oxygen 
concentration was measured during a series of discharges with Li-pellet injection. Lithium wall conditioning was used in a 1995 campaign evaluating high performance negative central shear (NCS) discharges. During this campaign, the highest values of stored energy, neutron rate, and $n_{i} T_{i} r_{E}$ achieved to date in DIII-D were obtained. High performance NCS discharges were also achieved prior to beginning lithium conditioning, but it is clear that shot reproducibility and performance were improved by lithium conditioning.

Li-pellet injection into Alcator $\mathrm{C}$-mod with molybdenum walls did not lead to a significant improvement of performance [146].

Laboratory experiments [147] show gettering of molecular oxygen and hydrogen atoms by $\mathrm{Li}$. This may be related to the observed reduction of the recycling flux in TFTR and to the decrease of the oxygen concentration in DIII-D. A strong temperature dependence of the hydrogen gettering can be expected due to the high vapor pressure of $\mathrm{H}$ over $\mathrm{LiH}$ at elevated temperature.

\section{Wall Conditioning in Future Devices}

It is not clear at present what the needs are for wall conditioning in future large tokamak or stellarator devices. Their very long pulse lengths will exhaust the transient effects which are used today to improve the plasma performance. Thin films on the high heat flux components will be eroded within a single discharge. It appears that the design and engineering of the devices will have to guarantee most of the impurity and recycling control during the stable long flat top phase. The start-up and ramp down phases are critical however. Problem areas may be the increase in plasma current and the incomplete confinement of particles during that phase, the low density which does not allow the divertor to work properly, the gradual temperature increase of the components, etc. The impurities released during the start-up may prevent reaching stable plasma conditions for ignition and impede the achievement of $\mathrm{H}$-modes. Hydrogen stored in the wall components from previous discharges may outgas due to the heating of the components and make density control impossible. Another important problem area is the need for clean up of tritium from in vessel components before a maintenance activity and the control of the T inventory in the whole device. Appropriate conditioning techniques appear to be indispensible.

The long pulse lengths of the next generation of devices implies that superconducting magnets will be used. The magnetic field will be maintained for long times to avoid cycling the coils. Thus wall conditioning techniques will have to be compatible with magnetic fields. Glow discharges are not suited under these circumstances. Pulse- and Taylor discharge cleaning are 
not adequate due to the fatigue of the components.

ECR- and ICR based techniques appear to be attractive in this respect. Both are not only compatible but need magnetic fields to couple energy into a plasma. The future machines will most likely be equipped with ICR and ECR heating systems which could be used in a downgraded operation mode at low power to produce cleaning plasmas. ECR cleaning at $2.45 \mathrm{GHz}$ in hydrogen is a established technique. First promising results on the ECR-based boronizaiton were recently obtained on Alcator C-Mod [148]. However, the ECR based deposition of thin films in a toroidal geometry with magnetic field has not been studied systematically yet. The same holds for ICR based techniques. Both are in their infancies of development. The discharge inherent techniques, discussed in section 5.5 , are probably candidates for maintaining good wall conditions during the discharges. Also here further research and development is required.

\section{Conclusions and Outlook}

Wall conditioning has played and is continuing to play an essential role on the way of controlled nuclear fusion research towards ignition. Many of the advances in controlled nuclear fusion have been closely linked to progress in our abilities to control the impurity influx and the hydrogen recycling.

Devices with metal walls largely rely on discharge cleaning techniques in hydrogen gas like pulse cleaning, Taylor discharge cleaning, glow- or ECR discharge cleaning. The key mechanism is the conversion of surface contaminants into volatile species by reactions with atomic hydrogen. Often these techniques were used in conjunction with gettering by $\mathrm{Ti}$ or $\mathrm{Cr}$.

Present large devices have a major part of their wall area covered with the low-Z material graphite. Helium glow discharge conditioning has been very successful in these cases both for removing desorbable impurities and for controlling the hydrogen recycling. In most machines low- $Z$ wall coatings are used in addition (boronization, beryllium evaporation, siliconization). The latter have been instrumental for obtaining high performance plasmas. They have often induced or improved high confinement regimes. Gettering of oxygen is a key mechanism in reducing the low-Z impurity content $(0, C)$ of the plasma. Wall pumping is the important mechanism in reducing the flux of hydrogenic neutral particles in most of the present devices. The effects are transient in all cases. They saturate beyond a certain hydrogen fluence and will not be available for particle control in steady state. Active external pumping by pump limiters and pumped divertors may keep the hydrogen concentration of the wall below the saturation limit and may thus, in an 
indirect way, provide some wall pumping in long pulse devices during transient phases of the discharge.

Thin wall coatings will be rather rapidly eroded or buried by codeposited material in future long pulse devices. In situ techniques for replenishment as are plasma fueling by reactive gases or pellets on in situ plasma spraying of components between discharges will have to be developed. Wall coatings will probably be an important option for future devices to provide and maintain clean plasmas up to the burn phase of the discharge until other means, like a gas target divertor, are fully operative.

\section{Acknowledgement}

The author greatfully acknowledges fruitful discussions with H.G. Esser, V. Philipps, and G. Jackson. 


\section{REFERENCES}

[1] G.M. McCracken and P.E. Stott, Nucl. Fusion 19 (1979), 889

[2] "Physics of Plasma-Wall-Interaction in Controlled Fusion Devices", D.E. Post, R. Behrisch (Eds), NATO AISI series, Plenum Press, New York, London, 1986

[3] P.C. Stangeby and G.M. McCracken Nucl. Fusion 30 (1990), 1225

[4] Physical Processes of the Interaction of Fusion Plasmas with Solids, W. Hofer, J. Roth (eds.), Academic Press Inc., San Diego (1996)

[5] S.I. Itoh, K. Itoh and A. Fukayama, J. Nucl. Mater. 220-222, (1995) 117

[6] D. Reiter, P. Bogen, U. Samm, J. Nucl. Materials 196-198 (1992), 1059

[7] A.M. Messiaen, J. Ongena, U. Samm et al., Nucl. Fusion 34, (1994), 825

[8] J. Neuhauser, M. Alexander, G. Becker, et al., Plasma Phys. Contr. Fusion 37 (1995) A37

[9] J. Winter et al., Phys. Rev. Lett. 71 (1993), 1549

[10] G.F. Matthews, D. Elder, G.M. McCracken et al., J. Nucl. Mater. 196-198 (1992), 253

[11] A. Refke, E. Vietzke, V. Philipps et al., to be published

[12] E. Vietzke, A. Refke, V. Philipps and M. Hennes, J. Nucl. Mater 220222 (1995), 249

[13] V. Philipps, E. Vietzke, M. Erdweg et al., Plasma Phys. Contr. Fusion 31 (1989), 1685

[14] K.L. Wilson, R.A. Causey, W.L. Hsu et al., J. Vac. Sci. Techn. A8 (1990), 1750

[15] K.J. Dietz, F. Waelbroeck and P. Wienhold, JÜL-IPP 1448 (1977)

[16] F.G. Waelbroeck, T. Banno, H.G. Esser et al., J. Nucl. Mater. 162164 (1989) 496

[17] F. Waelbroeck, P. Wienhold, J. Winter, J. Nucl. Mater. $111+112$ (1982), 185

[18] B.L. Doyle, J. Nucl. Mater. $111+112$ (1982), 628

[19] J. Winter, F.G. Waelbroeck, P. Wienhold et al., J. Vac. Sci. Techn. A2 (1984), 79

[20] K.L. Wilson, J. Nucl. Mater. $103+104$ (1981), 453

[21] P. Wienhold, M. Profant, F. Waelbroeck and J. Winter,

J. Nucl. Mater. $93+94$ (1980), 866

[22] P. Wienhold, M. Profant, F. Waelbroeck and J. Winter, Report JÜL-1825, 1983, Forschungszentrum Jülich GmbH, Germany

[23] M.I. Baskes, DIFFUSE 83, SAND 83-8231

[24] G. Saibene, R. Sartori, A. Tanga et al., J. Nucl. Mater. $176+177$ (1990), 618 
[25] J. Ehrenberg in Ref. [4]

[26] J. Ehrenberg, J. Nucl. Mater. 162-164 (1989), 63

[27] W. Möller, J. Nucl. Mater. 162-164 (1989),138

[28] J. Pillath, J. Winter and F. Waelbroeck, J. Nucl. Mater. 162-164 (1989), 1046

[29] C. Grisolia, L.D. Horton, and J. Ehrenberg, J. Nucl. Mater. 220-222 (1995), 516

[30] J. Winter, J. Vac. Sci. Techn. A5 (1987), 2286

[31] G.L. Jackson, T.S. Taylor and P.I. Petersen, in "Surface Conditioning of Vacuum Systems", Am. Vac. Soc. Series 8, Conf. Proc. Nr. 199, p 39

[32] J. Winter, Plasma Phys. Contr. Fusion 30 (1994), 13263

[33] J. Winter, J. Nucl. Mater 161, 265(1989)

[34] K.H. Dippel, K.H. Finken, G.J. Thomas et al., Proc. 12th EPS Contr. Fusion and Plasma Physics, Budapest, 1985, 9F11 - 531

[35] T. Loarer, A. Grosman, M. Chatelier et al., J. Nucl. Mater. 220-222 (1995), 183

[36] M.A. Mahdavi, S.L. Allen, D.R. Baker et al., J. Nucl. Mater. 220-222 (1995), 13

[37] JET divertor pumping

[38] P.K. Mioduszewski, J.P. Hogan et al., J. Nucl. Mater. 220-222, (1995), 91

[39] H.F. Dylla et al., J. Vac. Sci Techn 17 (1980), 286

[40] F.G. Waelbroeck, J. Winter, P. Wienhold et al., Proc. IX Int. Vac. Congress - V. Int. Conf. Solid Surfaces, Madrid (19803), p. 693

[41] Y. Sakamoto, Proc. IX Int. Vac. Congress - V. Int. Conf. Solid Surfaces, Madrid (1983), p. 716

[42] J. Burt, G.M. McCracken and P.E. Stott, in:

Proc. Int. Symp. on Plasma-Wall-Interactions, Jülich, 1976 (Pergamon, Oxfordshire, 1977), p. 457

[43] TFTR-Group, in:

Proc. Int. Symp. on Plasma-Wall-Interactions, Jülich, 1976 (Pergamon, Oxfordshire, 1977), p. 457

[44] L. Oren, and R.J. Taylor, Nucl. Fusion 17 (1977) 1143

[45] H.F. Dylla, J. Nucl. Mater. $93+94$ (1980) 61

[46] F.G. Waelbroeck, J. Winter and P. Wienhold J. Vac. Sci. Techn. A2 (1984), 1521

[47] J. Winter, F. Waelbroeck, P. Wienhold et al., J. Nucl. Mater. $128+129$ (1984) 841

[48] H.F. Dylla, W.R. Blanchard, R.B. Krawchuk et al., J. Vac. Sci. Techn. A2 (1984), 1188

[49] Y. Sakamoto, S. Ishii, K. Yano et al.,

Fusion Technology 1982, Pergamon Press, vol. 1, p. 347 
[50] Ph. Staib, H.F. Dylla, and S.M. Rossnagel, J. Vac. Sci. Techn. 17 (1980) 291

[51] R.E. Clausing, K.G. Tschersich, H.P. Fleischhauer et al., J. Nucl. Mater $122+123$ (1984), 1145

[52] R.E. Clausing and L. Heatherly, J. Nucl. Mater. $\underline{128+129}$ (1984), 855

[53] D. Cowgill, W. Hsu, private communication

[54] H.F. Dylla, in "Surface Conditioning of Vacuum Systems", Am. Vac. Soc. Series 8, Conf. Proc. Nr. 199, p. 3

[55] G.L. Jackson, T.S. Taylor and P.L. Taylor, Nucl. Fusion $\underline{30}$ (1990), 2305

[56] J. Pillath, Rep. JÜL 2292 (1989), Forschungszentrum Jülich GmbH

[57] G.L. Jackson, J. Vac. Sci. Techn. A10 (1992), 1244

[58] G.L. Jackson, D.R. Baker, K.L. Holtrop et al., J. Nucl. Mater. 220$\underline{222}$ (1995), 173

[59] K.L. Holtrop, G.L. Jackson, A.G. Kellman et al., J. Vac. Si. Techn. A12 (1994), 1269

[60] TFR Group, J. Nucl. Mater $76+77$ (1978), 587

[61] H.F. Dylla et al., J. Vac. Sci. Techn. 16 (1979), 752

[62] J.E. Simpkins, W.A. Gabbard, L.C. Emerson and P.K. Mioduszewski, J. Nucl. Mater $122+123$ (1984), 148

[63] J.E. Simpinks, P. Mioduszewski and L.W. Stratton, J. Nucl. Mater $111+112$ (1982), 827

[64] J. Simpkins, L.C. Emerson, P. Mioduszewski and L.W. Stratton J. Vac. Sci. Technol. A1 (1983), 1306

[65] A. Peacock et al., Report JET-R (90) 02, 1990

[66] R.J. Taylor, R.F. Bunshah and F. Schwirzke, J. Nucl. Mater. $93+94,(1980), 338$

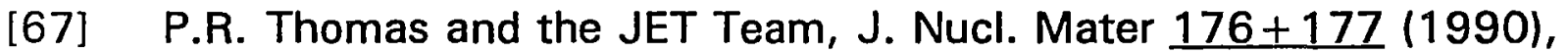
3

[68] J. Winter, J. Nucl. Mater. 145-147 (1987), 131

[69] J. Winter, H.G. Esser, L. Könen et al., J. Nucl. Mater. 162-164 (1989), 713

[70] U. Samm, P. Bogen, H.G. Esser et al., J. Nucl. Mater. 220-222 (1995), 25

[71] J. v. Seggern, J. Winter, L. Grobusch et al., J. Nucl. Mater. 220-222 (1995), 677

[72] H.G. Esser, H.B. Reimer, J. Winter, and D. Ringer, Fusion Technology 1988, Vol. 1, p. 791

[73] J. Winter, H.G. Esser, H. Reimer et al., J. Nucl. Mater. $176+177$ (1990), 486

[74] H.G. Esser, A.A.M. Oomens "Boronization of RTP", private communication 
[75] C. Boucher, F. Martin, B.L. Stomsfield et al., J. Nucl. Mater. 196198 (1992), 587

[76] M. Saidoh et al., Jpn. J. Appl. Phys. $\underline{32}$ (1993) 3276

[77] V.Kh. Alimov, D.B. Bogomolov, M.N. Churaeva et al., J. Nucl. Mater. 196-198 (1992), 670

[78] V.A. Sharapov, S.V. Mirnow, S.A. Grashin et al., J. Nucl. Mater. 220-222 (1995), 730

[79] F.L. Tabarés, E. de la Cal, D. Tafalla et al., J. Nucl. Mater. 220-222 (1995), 688

[80] P. Karduck, N. Amman, H.G. Esser, and J. Winter, Fresenius Chem. J. Anal. 341 (1991), 315

[81] G.L. Jackson, J. Winter, K.H. Burrell et al., J. Nucl. Mater. 196-198 (1992), 236

[82] J. Winter et al., Nucl. Instr. and Meth. B23 (1987) 538

[83] G. Kögel, D. Schödlbauer, W. Triftshäuser and J. Winter, J. Nucl. Mater. 162-164 (1989) 1550

[84] G. Kögel, D. Schödlbauer, W. Triftshäuser and J. Winter, J. Nucl. Mater. 162-164 (1989), 876

[85] J. Dünnwald, J. Winter, E. Vietzke and A. Otto, Appl. Phys. A46 (1988) 91

[86] J. Seggern, P. Wienhold, H.G. Esser et al., J. Nucl. Mater. 176-177 (1990), 357

[87] S. Veprék, S. Rambert, M. Heintze et al., J. Nucl. Mater. 162-164 (1989), 724

[88] E. Vietzke, V. Philipps, K. Flaskamp, J. Winter and S. Veprék, J. Nucl. Mater. $176+177$ (1990) 48

[89] A. Schenk, B. Winter, C. Lutterloh et al., J. Nucl. Mater. 220-222 (1995), 767

[90] M. Yamage, H. Sugai, T. Saito et al., J. Nucl. Mater. 220-222 (1995) 743

[91] G. Kögel, Universität der Bundeswehr, Neubiberg, Germany, private communication

[92] R. Zehringer, H. Künzli, P. Oelhafen and Ch. Hollenstein, J. Nucl. Mater. $176+177$ (1990), 370

[93] J. Winter, J. Nucl. Mater. $176+177$ (1990), 14

[94] W. Beyer and H. Mell in "Disordered Semiconductors", M.A. Kastner, G.A. Thomas, and S.R. Ovshinsky (eds.), Plenum Press, New York, 1987, p. 641

[95] J.C. Angus, P. Koidl, and S. Domitz in: Plasma Deposited Thin Films, J. Mort., F. Jensen (eds.), CRC Press, Boca Raton, USA, 1986, p. 89

[96] M.L. Apicella, R. Bartiromo, G. Mazzitelli et al., Proc. 21 st EPS Conf. Contr. Fusion and Plasma Phys., Montpellier, 1994, Vol. II, p. 818 
[97] H.G. Esser, J. Winter, V. Philipps et al., J. Nucl. Mater. 196-198 (1992), 231

[98] H.G. Esser, V. Philipps, P. Wienhold et al., J. Nucl. Fusion $\underline{32}$ (1992), 278

[99] H.G. Esser, Stresa paper

[100] G.L. Jackson, J. Winter et al., Bull. Am. Soc. 38 (1993) 2062

[101] V. Philipps, A. Pospieszczyk, U. Samm et al., J. Nucl. Mater. 196198 (1992), 1106

[102] H.W. Kugel, J. Timberlake, R. Bell et al., J. Nucl. Mater. 220-222 (1995), 636

[103] D.J. Den Hartog and R.D. Kendrick, J. Nucl. Mater. 220-222 (1995), 631

[104] J.L. Terry et al., Plasma Phys. and Controlled Nuclear Fusion Research 1988 (IAEA, Vienna, 1989) Vol. 1, p. 27

[105] G. Staudenmaier, Ph. Staib, and G. Venus, J. Nucl. Mater. $\underline{76+77}$ (1978), 445

[106] H.F. Dylla et al., J. Vac. Sci. Technol. 16 (1979), 752

[107] Y. Gomay et al., J. Va. Sci. Technol. 16 (1979), 918

[108] Y. Gomay, T. Tazima and N. Fujisawa, J. Vac. Sci. Technol. 15 (1978), 103

[109] W. Poschenrieder, G. Staudenmaier, and Ph. Staib, J. Nucl. Mater. $93+94$ (1980), 823

[110] H.F. Dylla, W.R. Blanchard, R.B. Krawchuk et al., J. Vac. Sci. Techn. A2 (1984), 1188

[111] H.F. Dylla, W.R. Blanchard, R.J. Hawryluk et al., J. Nucl. Mater. $128+129(1984), 861$

[112] K.J. Dietz, D. Bartlett, G. Bäumel et al., J. Nucl. Mater. $\underline{128+129}$ (1984), 10

[113] G. Saibene, A. Rossi, R.D. Mank et al., J. Nucl. Mater. 220-222 (1995), 617

[114] G.L. Jackson, R.E. Clausing, A.F. Lietzke et al., J. Vac. Sci. Techn. A1 (1983), 1861

[115] R.E. Clausing, F. Waelbroeck, J. Winter et al., J. Nucl. Mater. 121 (1984), 249

[116] G.L. Jackson, J. Winter, K.H. Burrell et al., Phys. Rev. Lett.

[117] P.E. Stott, C.C. Daughney and R.A. Ellis, Nucl. Fusion 15 (1975), 431

[118] S.J. Fielding, J. Hugill, G.M. McCracken et al., Nucl. Fusion 17 (1977), 1382

[119] R.J. Colchin, C.E. Bush, P.H. Edmonds et al., J. Nucl. Mater. $76+77$ (1978) 405

[120] K. Bol, V. Anurasalam, M. Bitter et al., Proc. Int. Conf. Plasma Physics and Controlled Fusion Research, IAEA 1979, Vol. 1, p. II 
[121] D. Meade, W. Arunasalam, C. Barnes et al., Proc. 9th Europ. Conf. on Plasma Physics and Controlled Fusion, Oxford, 1979, p. 91

[122] A.J. Wooton, P.H. Edmonds, R.C. Isler and P. Mioduszewski, J. Nucl. Mater. $111+112$ (1982), 479

[123] P.K. Mioduzewski, R.C. Isler, J.E. Simpkins et al., Nucl. Fusion 27 (1987), 195

[124] H.F. Dylla, P.H. LaMarche, W.R. Blanchard et al., J. Vac. Sci. Techn. A4 (1986), 1753

[125] G. Fußmann, the ASDEX team, the NI team, and the ICRH team, J. Nucl. Mater. 145-147 (1987), 96

[126] J.P. Coad, K.H. Behringer and K.J. Dietz, J. Nucl. Mater. 145-147 (1987) 747

[127] N. Noda et al., J. Nucl. Mater. $145-147$ (1987), 709

[128] G.L. Jackson, J. Winter, S. Lippmann et al., J. Nucl. Mater. $176+177(1990), 311$

[129] K. Uo et al., 11 the Int. Conf. Plasma Physics and Controlled Fusion Research, Kyoto, Japan, Nov. 1986, IAEA-CN-47/D-I-1

[130] G.H. Wolf, H.L. Bay, G. Bertschinger, et al., Plasma Phys. Contr. Fusion 28 (1986), 1413

[131] U. Schneider, W. Poschenrieder, M. Bessenroth-Weberpals et al., J. Nucl. Mater. $176+177$ (1990), 89

[132] Ch. Hollenstein, B.P. Duval, T. Dudok de Witt, J. Nucl. Mater. $176+177(1990), 343$

[133] H.F. Dylla, G. Bell, R.J. Hawryluk et al., J. Nucl. Mater. $176+177$ (1990), 337

[134] G. Jackson, J. Winter, K.H. Burrell et al., J. Nucl. Mater. 196-198 (1992), 236

[135] E. Gauthier, C. Grisolia, A. Grosman et al., J. Nucl. Materials 196198 (1992), 637

[136] R. Grigull, R. Behrisch, R. Brakel et al., J. Nucl. Materials 196-198 (1992) 101

[137] S. Shinohara et al., J. Phys. Soc. Japan 61 (1992) 3030

[138] P. Wienhold, M. Rubel, J. von Seggern et al., J. Nucl. Mater. 196-198 (1992) 647

[139] J.P. Coad et al., J. Nucl. Materials $176+177$ (1990), 145

[140] J. Ehrenberg, V. Philipps, L. DeKock et al., J. Nucl. Mater. $176+177$ (1990) 226

[141] T. Hino, Y. Yamauchi, T. Yamashina, J. Nucl. Mater 210 (1994) 226

[142] S. Higashijima, T. Sugie, H. Kubo et al., J. Nucl. Mater. 220-222 (1995) 375

[143] H.G. Esser, J. Winter et al., to be published

[144] J. Strachan, D.K. Mansfield, M.G. Bell, et al.

J. Nucl. Materials 217 (1994), 145

[145] G.L. Jackson, private communication 
[146] B. Lipschultz, private communication

[147] H. Sugai, H Toyoda, K. Nakamura et al., J. Nucl. Mater. 220-22 (1995), 259

[148] H.G. Esser, B. Lipschultz, private communication 
Figure Captions

Fig. 1 Radiation parameter $R$ of various plasma impurities as a function of $T_{e}$. The radiated power $P_{\text {rad }}$ is given by $P_{\text {rad }}=R \cdot n_{e} \cdot n_{i}$ where $n_{e}$ and $n_{i}$ are electron and impurity density, respectively.

Fig. 2 Intensity of the O-VI line in TEXTOR as a function of discharge number. Between discharges 494 and 495 a glow discharge in $\mathrm{He}$ was operated for 15 minutes.

Fig. 3 Schematic representation of the oxygen cycle in a tokamak with all carbon surfaces.

Fig. 4 Temperature dependence of the chemical reaction yields from graphite (top) and from $\mathrm{B}_{4} \mathrm{C}$ (bottom) upon $1.5 \mathrm{keV} \mathrm{O} \mathrm{O}^{+}$impact. $C$ (chem) and $C+B$ (chem), respectively, represent the total chemical erosion by oxygen.

Fig. 5 Temperature dependence of the equilibrium constant $\mathrm{K}_{\mathrm{p}}=\mathrm{p}\left(\mathrm{H}_{2} \mathrm{O}\right) / \mathrm{p}\left(\mathrm{H}_{2}\right)$ for the reduction of various metal oxides by molecular hydrogen (after [45]).

Fig. 6 Arrhenius diagram of the surface recombination rate constant $K_{r}$ from various laboratory and in situ tokamak measurements (after [19] with references for the various data points).

Fig. 7 Comparison of the experimental $D$ plasma inventory with the PERI simulation. $T_{w}=573 \mathrm{~K}, \mathrm{D}=7 \times 10^{-13} \mathrm{~m}^{2} \mathrm{~s}^{-1}, \mathrm{~K}_{\mathrm{r}}=2 \times 10^{-33}$ $\mathrm{m}^{4} \mathrm{~s}^{-1}$.

Fig. 8 Outgassing of the TEXTOR liner during a ramp heating from 200 ${ }^{\circ} \mathrm{C}$ to $400^{\circ} \mathrm{C}$. The liner had been carbonized at $200^{\circ} \mathrm{C}$. The inset shows the residual gas pattern at about $380^{\circ} \mathrm{C}$.

Fig. 9 Increase of partial pressures in the presence of the RG-discharges over the normal outgassing rates for the first cleaning run after a long opening of TEXTOR.

Fig. 10 Desorption of $\mathrm{CO}$ and $\mathrm{H}_{2}$ from the carbonized TEXTOR wall by a glow discharge in helium. 
Fig. 11 Helium and Argon induced desorption of hydrogen from saturated carbon films and from DIII-D graphite tiles as a function of incident ion energy normalized to a fluence of $1 \times 10^{16}$ ions $/ \mathrm{cm}^{2}$. Two data points for hydrogen saturated carbonization films are included (o He desorption, A Ar desorption).

Fig. 12 Sticking coefficients for $\mathrm{O}_{2}, \mathrm{~N}_{2}$, and $\mathrm{D}_{2}$ on $\mathrm{Ti}$ and $\mathrm{Cr}$ as a function of surface coverage of the gas on the metal surface.

Fig. 13 Schematic scetch of the arrangement for plasma assisted thin film deposition in TEXTOR.

Fig. 14 Sticking coefficient of oxygen ions on siliconized surfaces as a function of ion dose. The data for $\mathrm{a}-\mathrm{C} / \mathrm{Si}: \mathrm{H}$ are corrected for $\mathrm{CO}$ production.

Fig. 15 Daily average of central $Z_{\text {eff }}$ (from conductivity) in ohmic discharges in TEXTOR with all metal walls. Line averaged densities were $(1.5-2) \times 10^{13} \mathrm{~cm}^{-3}$. Conditioning actions are indicated in the figure.

Fig. 16 Radiated power, $\mathrm{Z}_{\text {eff }}$ and chromium influx as a function of the line averaged electron density at constant ohmic input power in the early phase of JET operation, after [112].

Fig. 17 Energy spectrum of the $B(1 \mathrm{~s})$ photoelectrons for redeposited layers from the boronized TEXTOR (top) and a boronization film with $B / C$ 1:1 (reference, lower part), measured by $x$-ray induced photoelectron spectroscopy. Most $B$ atoms of the redeposited film are bound to oxygen which has been gettered from the SOL plasma, whereas no oxygen contamination is observed in the reference film.

Fig. 18 Absolute oxygen fluxes at the TEXTOR main limiter as a function of line averaged electron density for boronized and siliconized wall conditions.

Fig. 19 Fraction $y$ of radiated power $P_{\text {rad }}$ (in \%) to the total input power $P_{\text {tot }}$ as a function of the line averaged central electron density for $1.5 \mathrm{MW}$ neutral beam heated discharges in the boronized and siliconized TEXTOR. 
Fig. 20 Wall pumping rate $\phi_{\mathrm{w}}$ in JET for two tokamak discharges with beryllium and carbon walls, respectively, after [140].

Fig. 21 Wall pumping rate $\phi_{\mathrm{w}}$ (a), $\mathrm{N}_{\text {tot }}$ during a discharge (b), and $\phi_{\mathrm{w}}$ at the end of the density flat top as a function of $N_{\text {tot }}$ for two consecutive discharge series (c) after one siliconization in TEXTOR.

Fig. 22 Rate of rise of the density increase normalized to the beam fueling rate $\Gamma_{\text {beam }}$ at the onset of beam heating in DIII-D with carbon walls and after boronization, as a function of the ohmic target plasma density. Low values correspond to low hydrogen recycling. Discharges with $\mathrm{H}$ mode confinement are achieved after boronization (Bzn) and helium glow wall conditioning (He GWC).

Fig. 23 Energy confinement time as a function of the recycling deuterium flux normalized to the electron density in JT 60-U before and after a fresh boronization.

Fig. 24 Evolution of the oxygen, carbon, and silicon fluxes normalized to the flux of deuterons measured at the poloidal limiter in TEXTOR as a function of discharge number. The fluxes were deduced from measured line intensities and the corresponding photon efficiencies.

Fig. 25 a) Erosion of boronization layers on the toroidal limiter of TEXTOR as exhibited by the decrease of the B II intensity for consecutive operation days.

b) Erosion of Be films evaporated onto the JET carbon limiters as exhibited by the Be/intensity as a function of discharge number. 
Table 1

Free energy of formation $\Delta G$ and free energy of formation per oxygen atom $\Delta G / O$ for various oxides (at $0^{\circ} \mathrm{C}$ )
Z atom oxide
$-\Delta \mathbf{G}[\mathbf{k J} / \mathbf{m o l e}]$
$-\Delta \mathrm{G} / \mathrm{O}[\mathrm{kJ} / \mathrm{mole}]$
low - Z - getters

$\begin{array}{rllrr}4 & \mathrm{Be} & \mathrm{BeO} & 581 & 581 \\ 5 & \mathrm{~B} & \mathrm{~B}_{2} \mathrm{O}_{3} & 1194 & 397\end{array}$

medium - Z - getters

$\begin{array}{rllrr}12 & \mathrm{Mg} & \mathrm{MgO} & 569 & 569 \\ 13 & \mathrm{Al} & \mathrm{Al}_{2} \mathrm{O}_{3} & 1582 & 527 \\ 14 & \mathrm{Si} & \mathrm{SiO}_{2} & 857 & 428\end{array}$

high - Z-getters

\begin{tabular}{rrrrr}
22 & $\mathrm{Ti}$ & $\mathrm{TiO}_{2}$ & 889 & 444 \\
73 & $\mathrm{Ta}$ & $\mathrm{Ta}_{2} \mathrm{O}_{5}$ & 1911 & 352 \\
\hline \hline
\end{tabular}

bad getters

\begin{tabular}{lllll}
74 & $\mathrm{~W}$ & $\mathrm{WO}_{3}$ & 764 & 254 \\
26 & $\mathrm{Fe}$ & $\mathrm{Fe}_{2} \mathrm{O}_{3}$ & 742 & 247 \\
28 & $\mathrm{Ni}$ & $\mathrm{NiO}$ & 212 & 212 \\
\hline
\end{tabular}

reference molecules

\begin{tabular}{llllr}
1 & $\mathrm{H}$ & $\mathrm{H}_{2} \mathrm{O}$ & 228 & 228 \\
6 & $\mathrm{C}$ & $\mathrm{CO}^{2}$ & 394 & 197 \\
& $\mathrm{CO}$ & 137 & 137 \\
\hline & $\mathrm{Li}$ & $\mathrm{Li}_{2} \mathrm{O}$ & 134 & 134
\end{tabular}




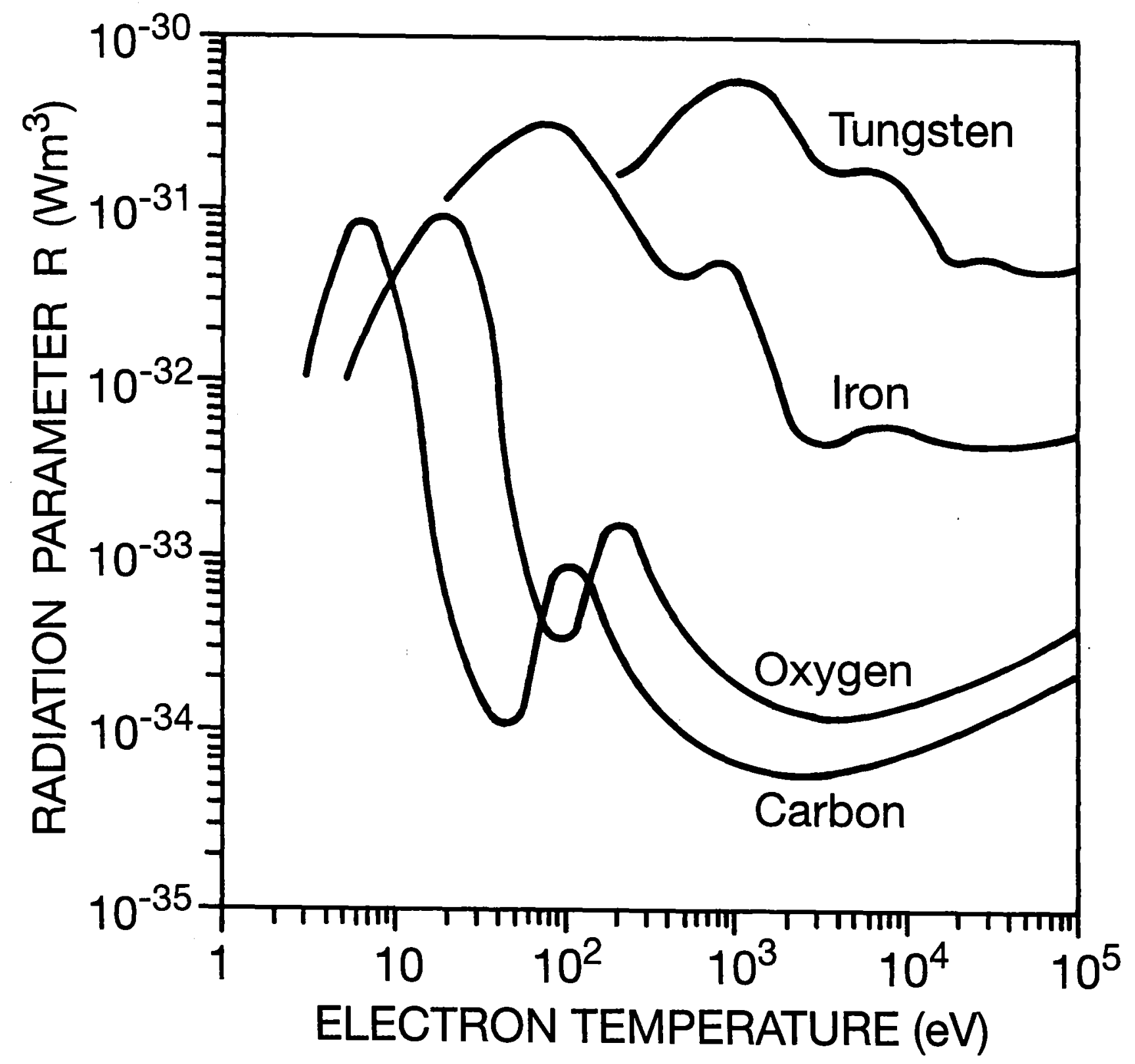




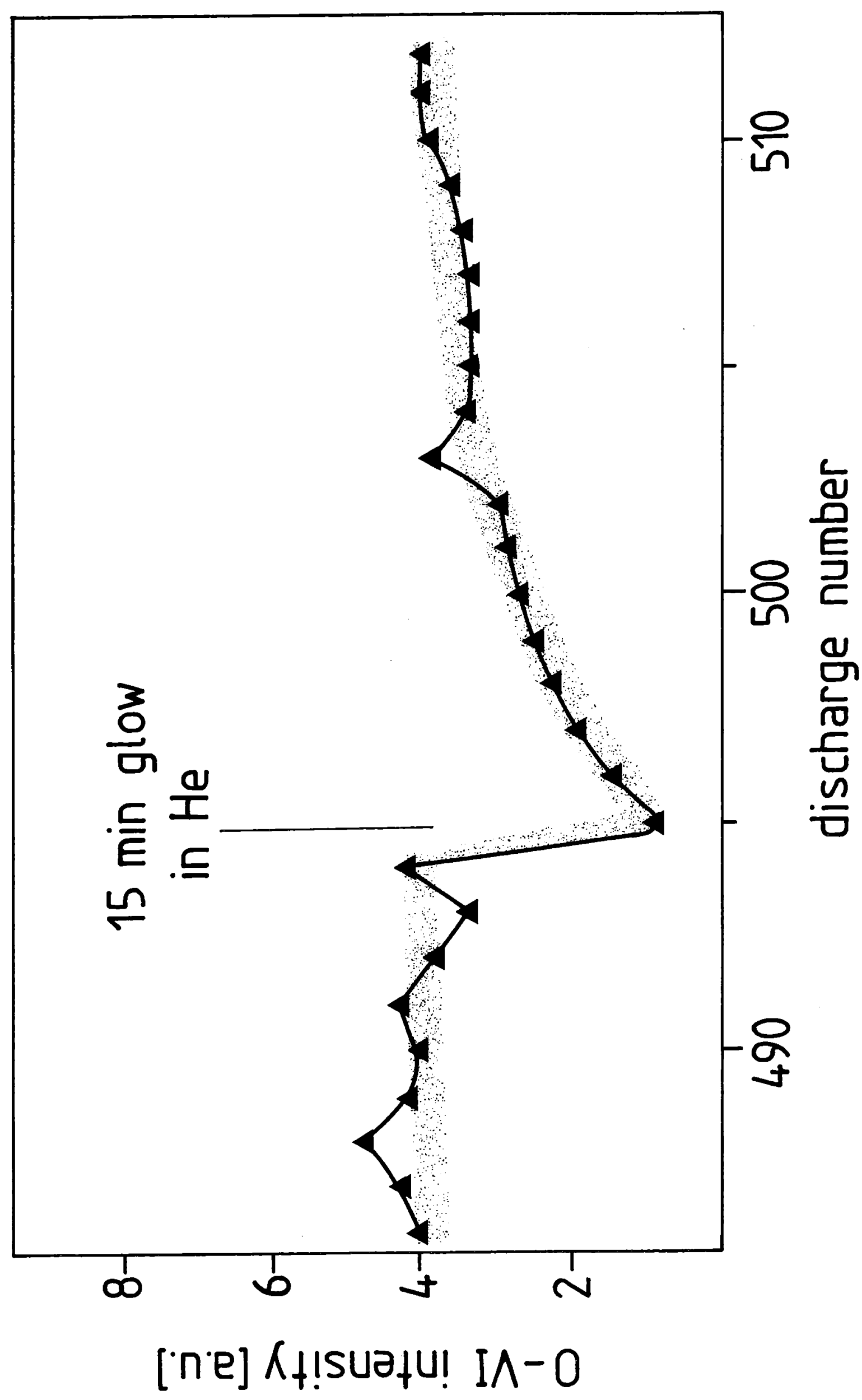




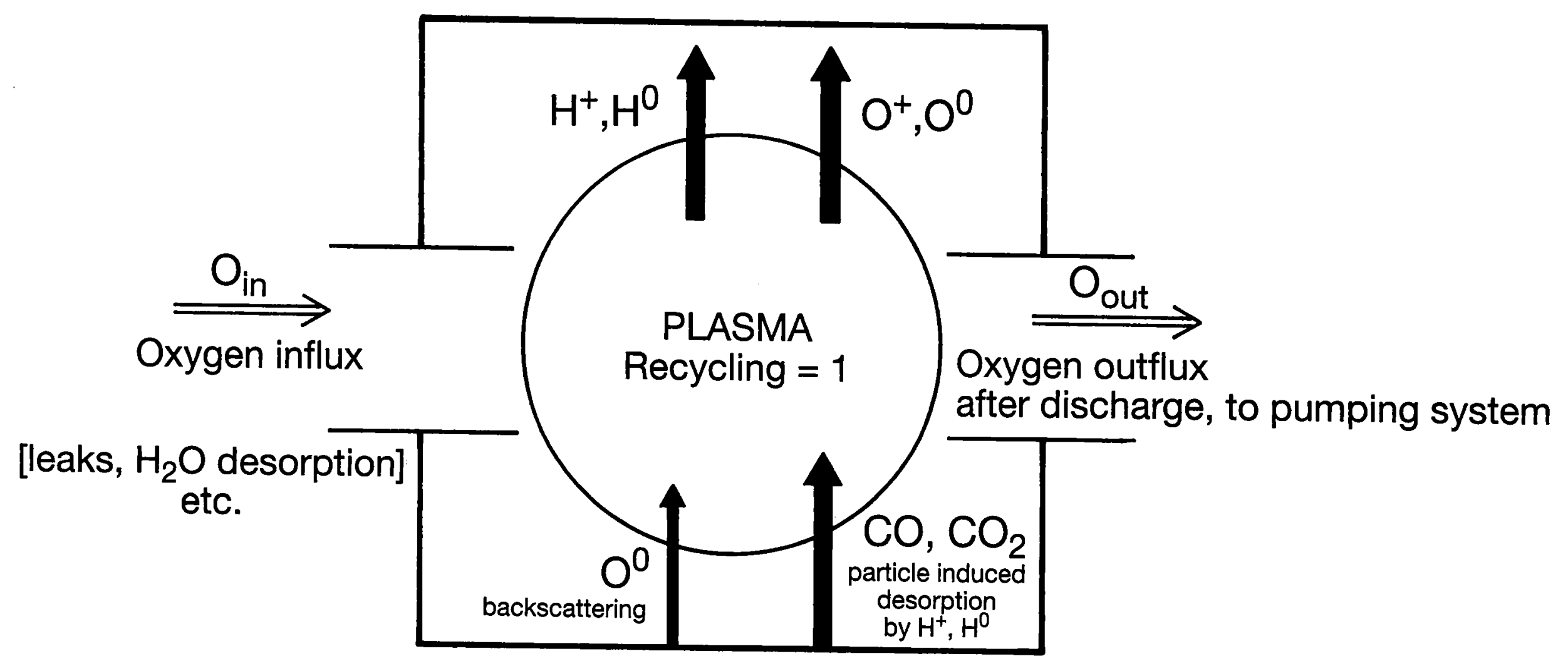




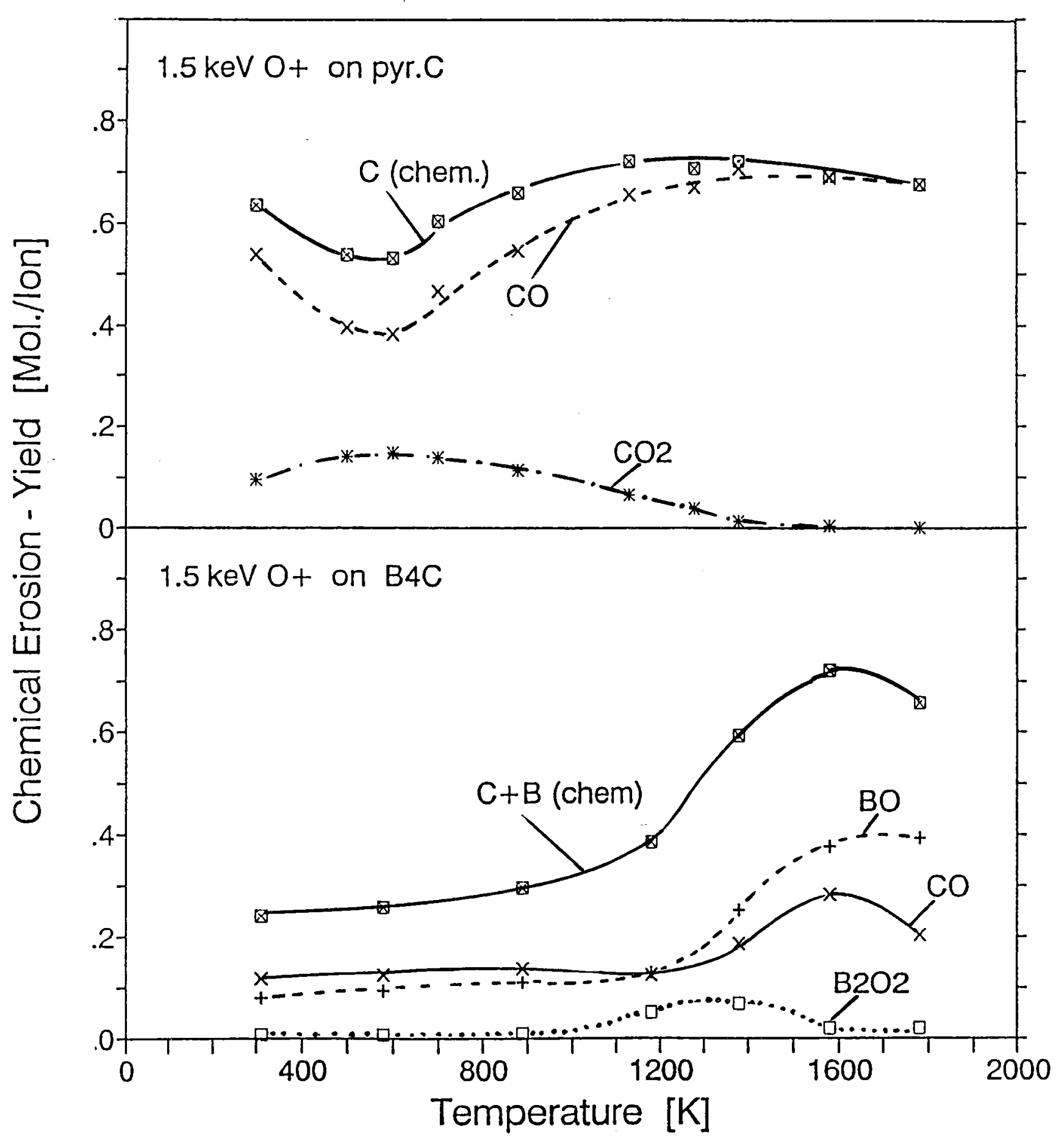




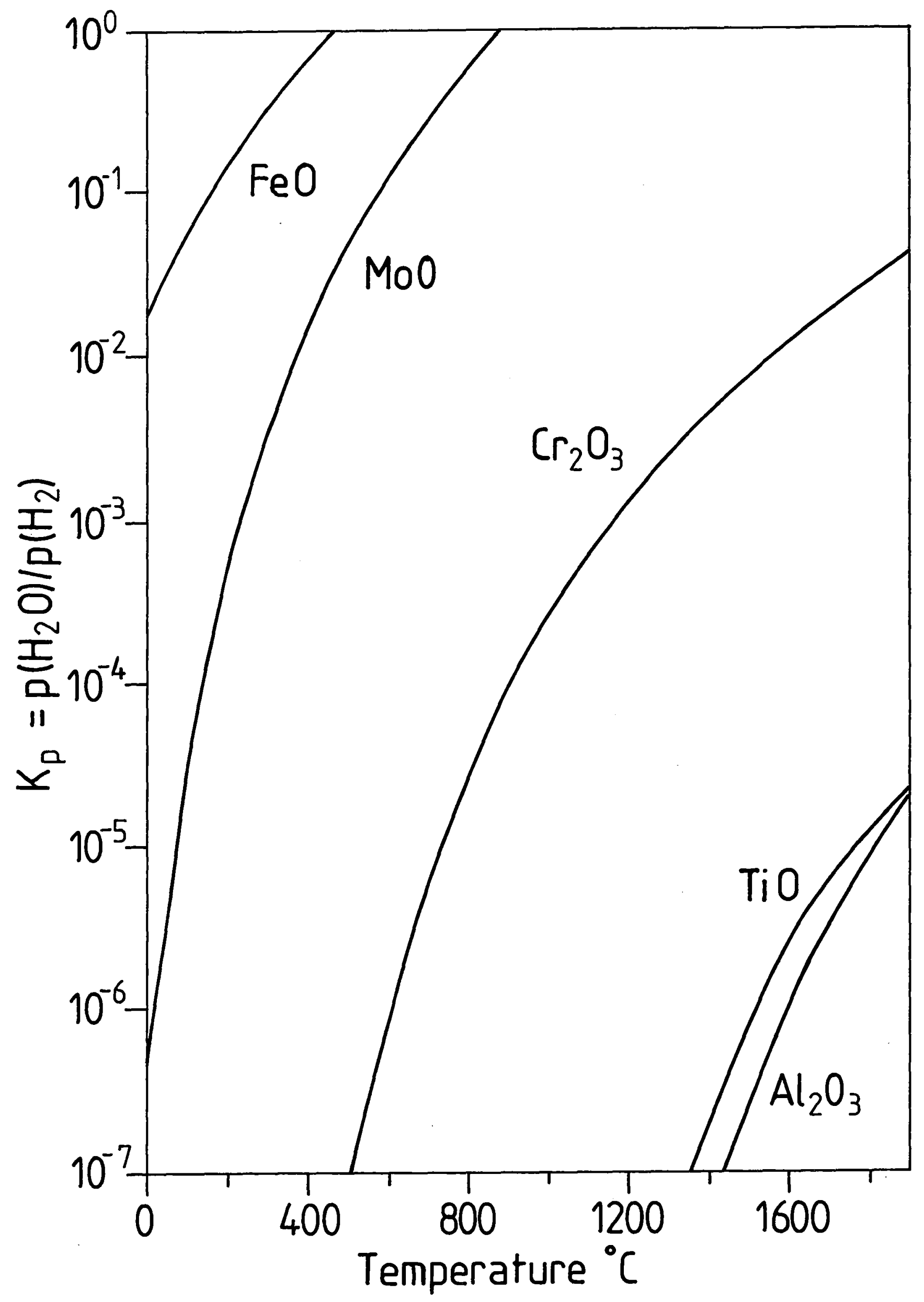




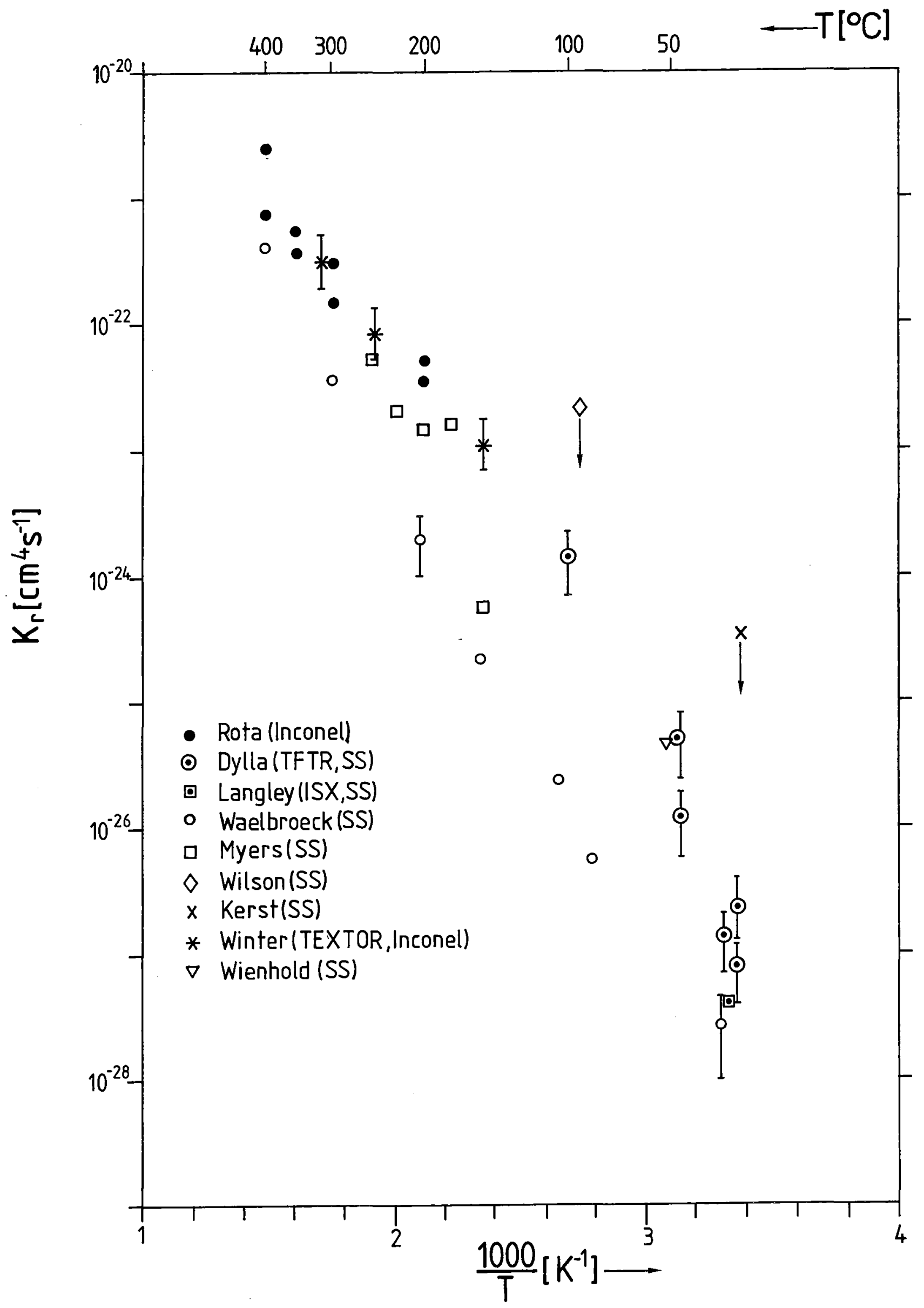


Fig 7

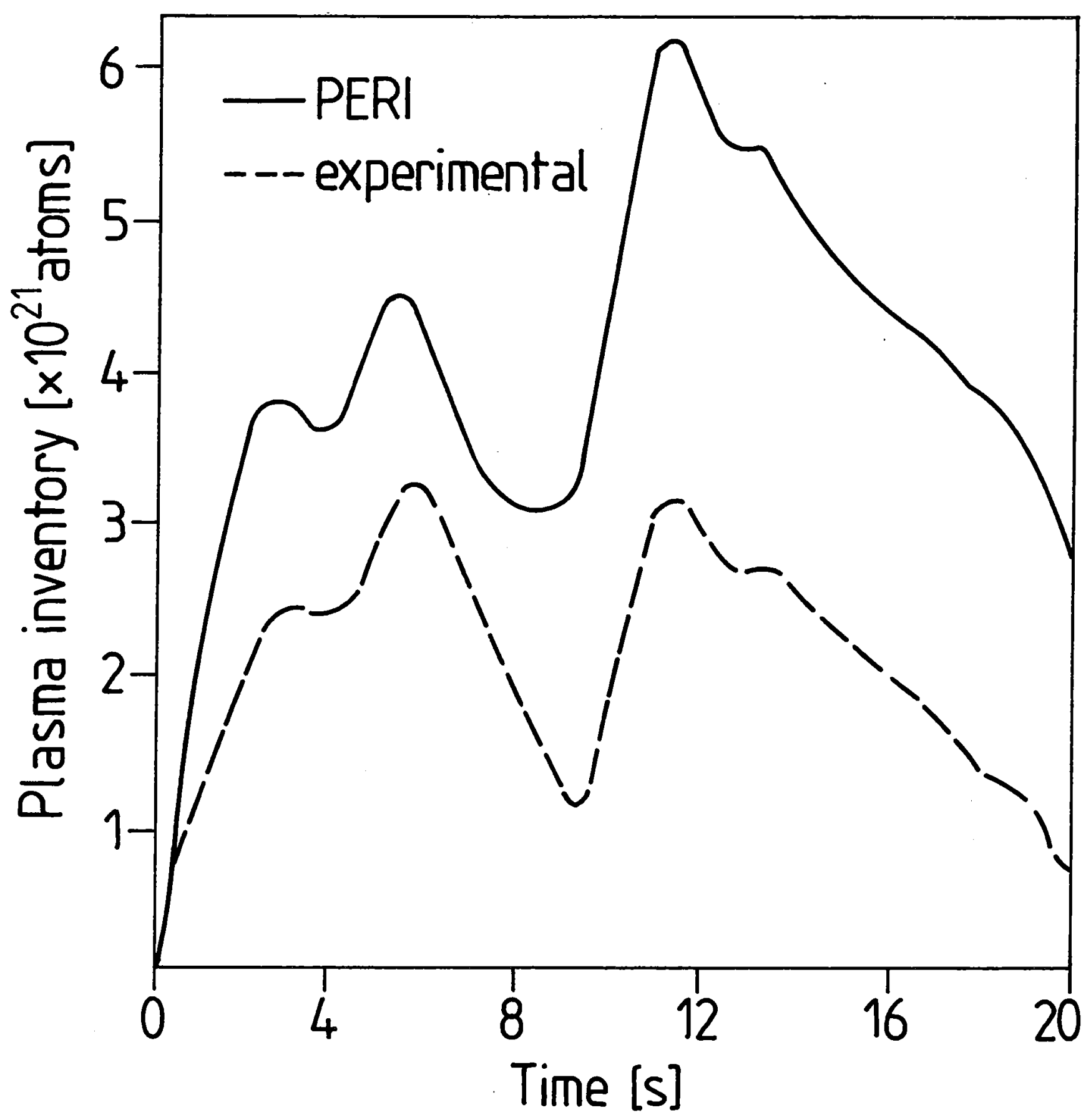



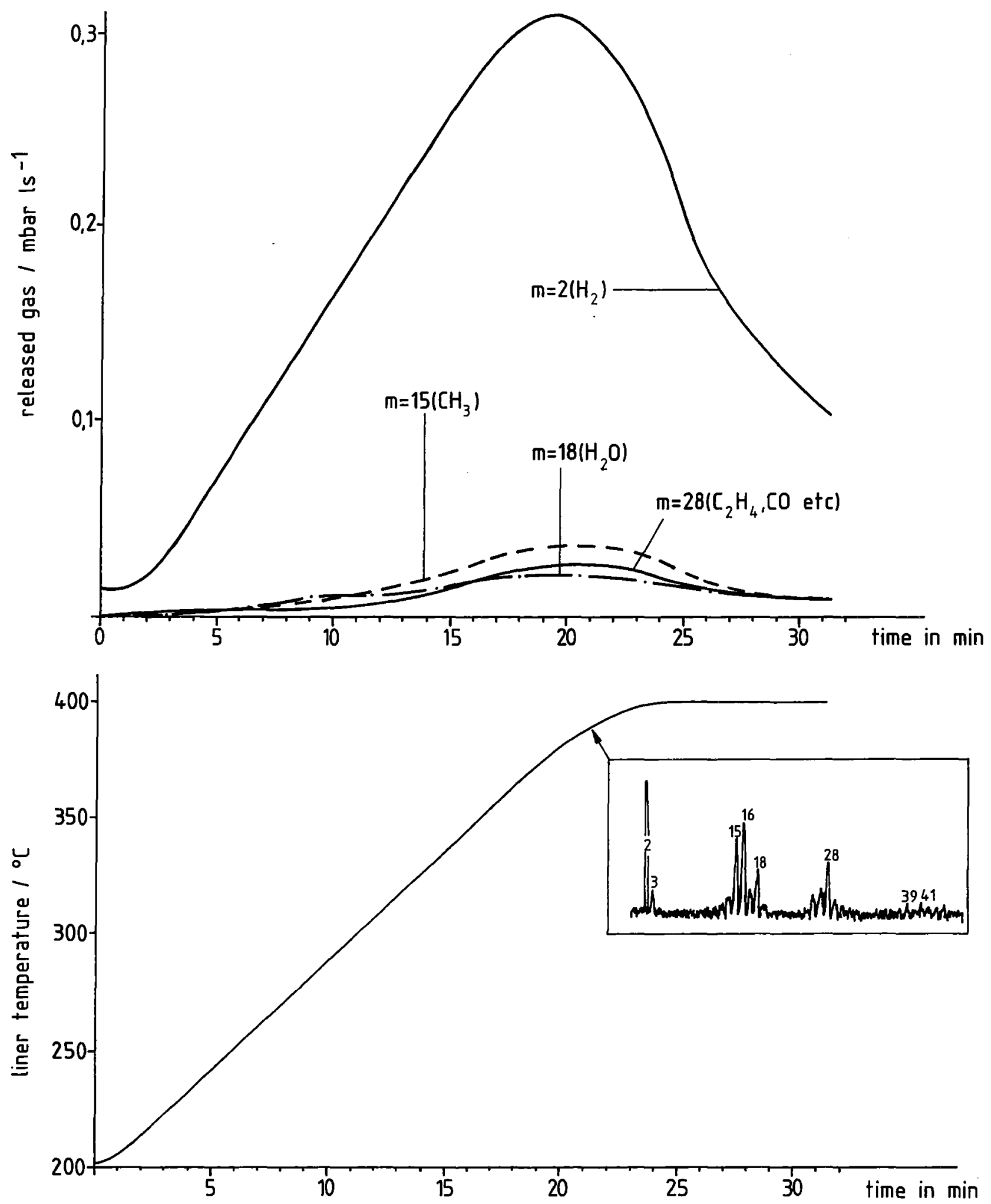

Ramp-heating of the carbonized TEXTOR liner from $200^{\circ} \mathrm{C}$ to $400^{\circ} \mathrm{C}$.

Released gas amount (upper curve) and temperature vs time correlation (lower curve). The insert shows a full mass spectrum taken at $390^{\circ} \mathrm{C}$. 
F栵.

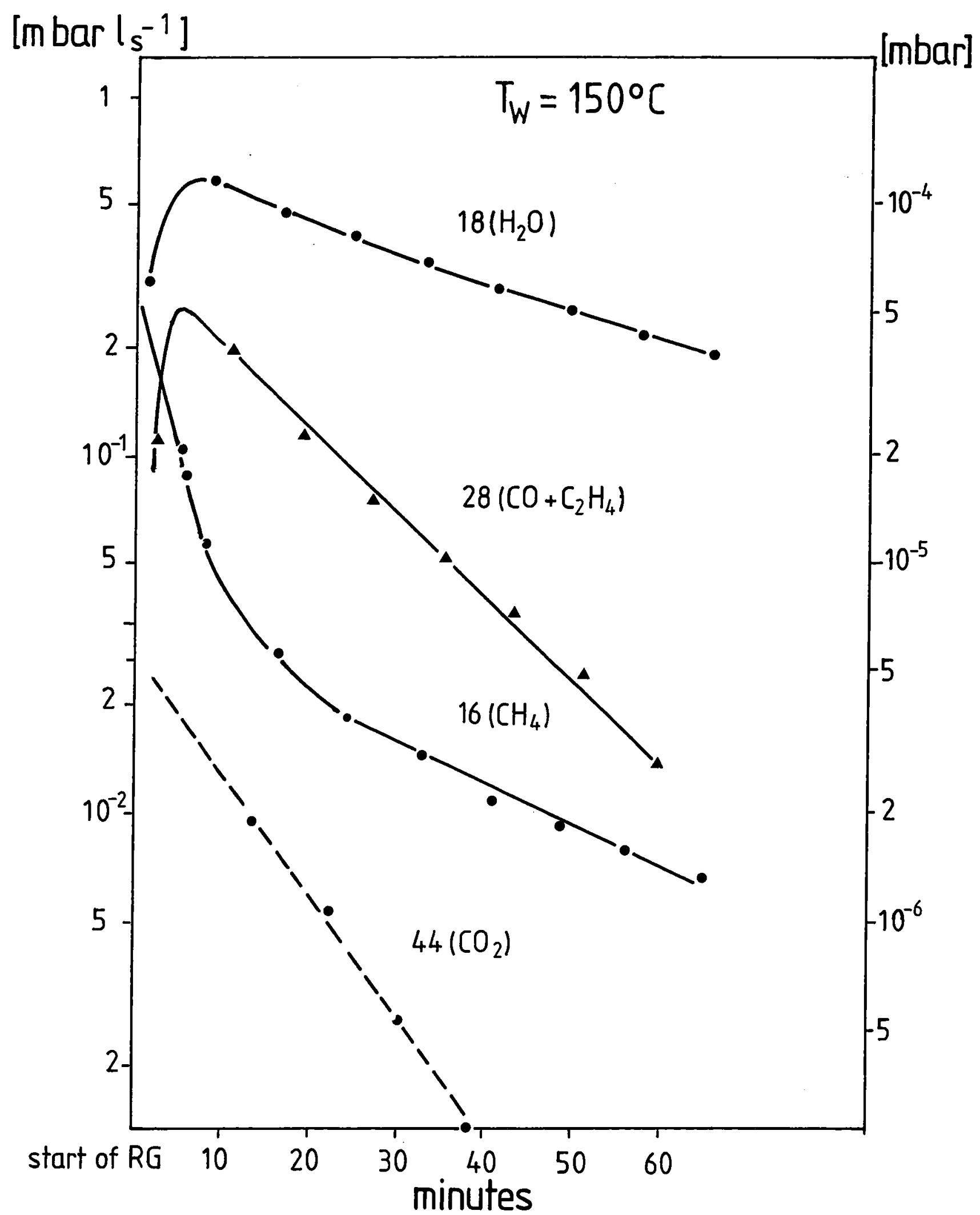




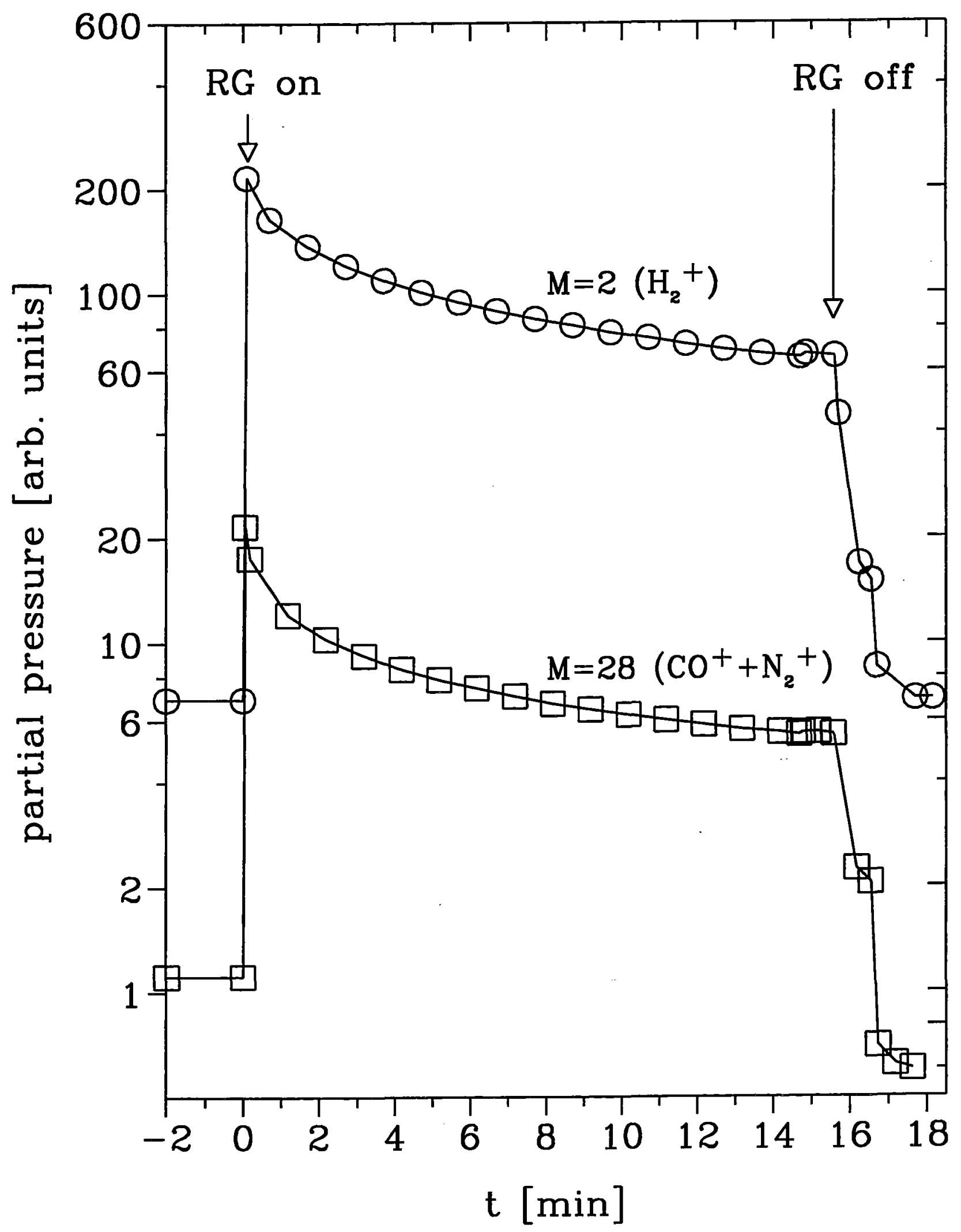


Fig. II

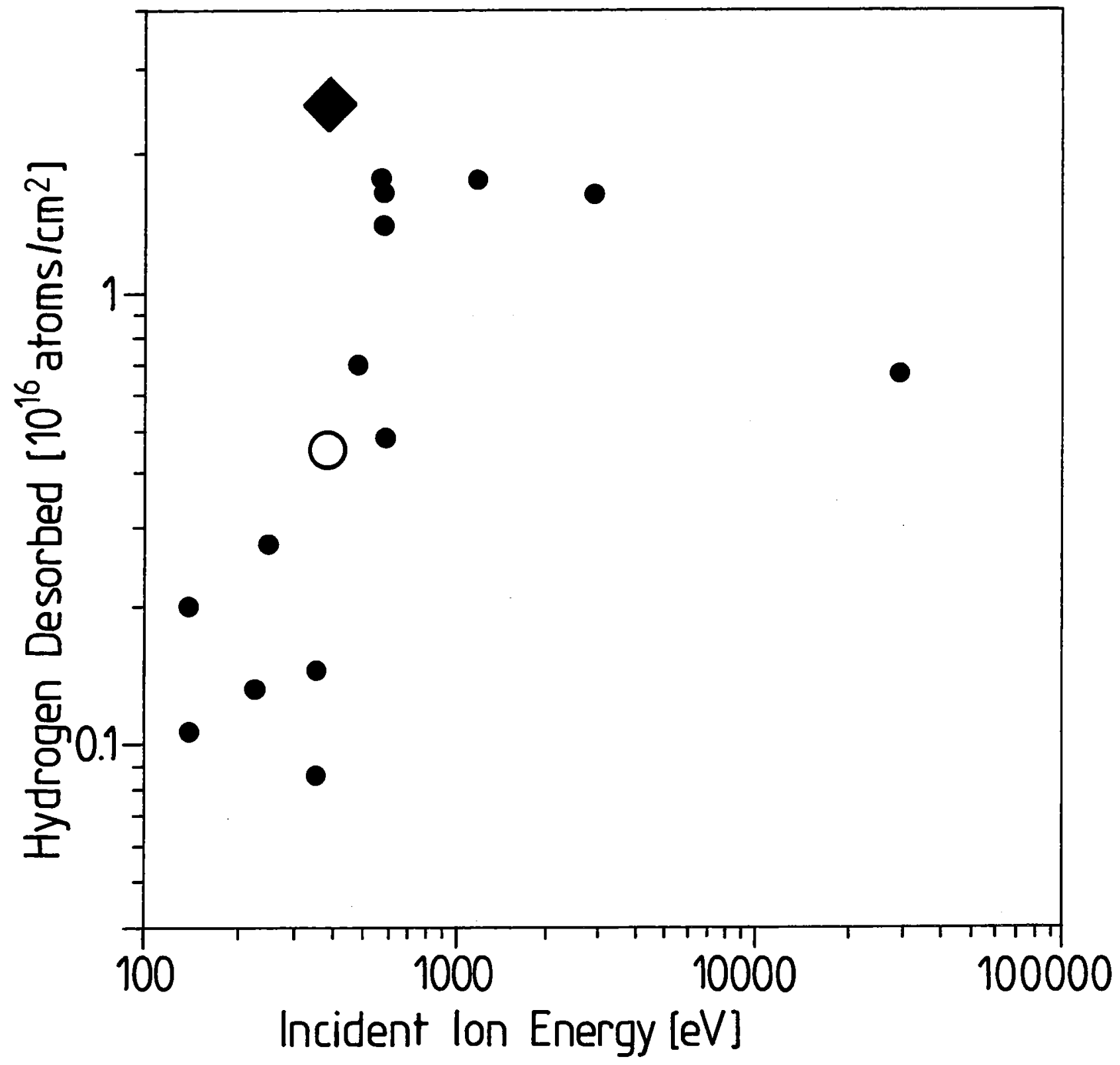




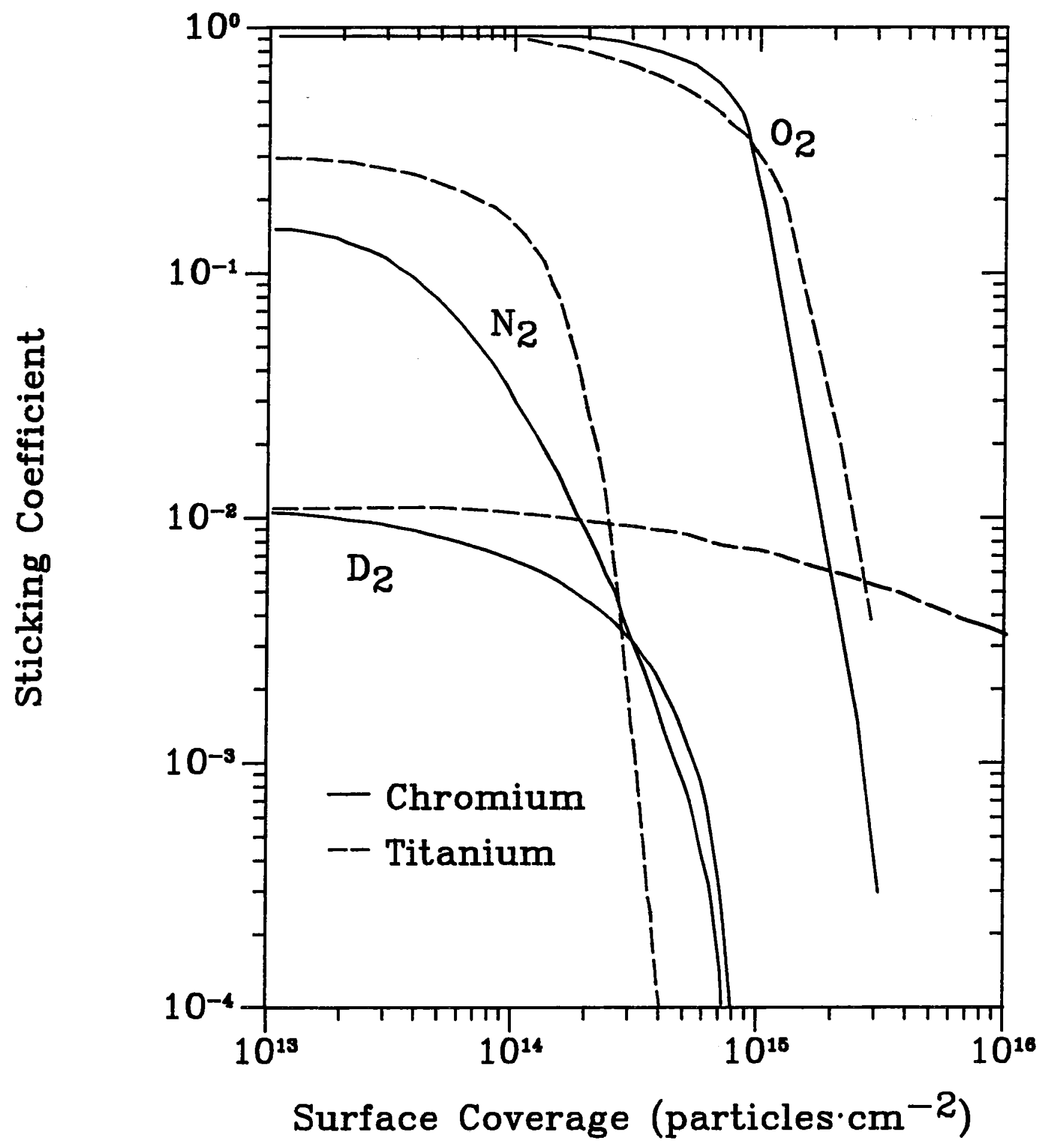




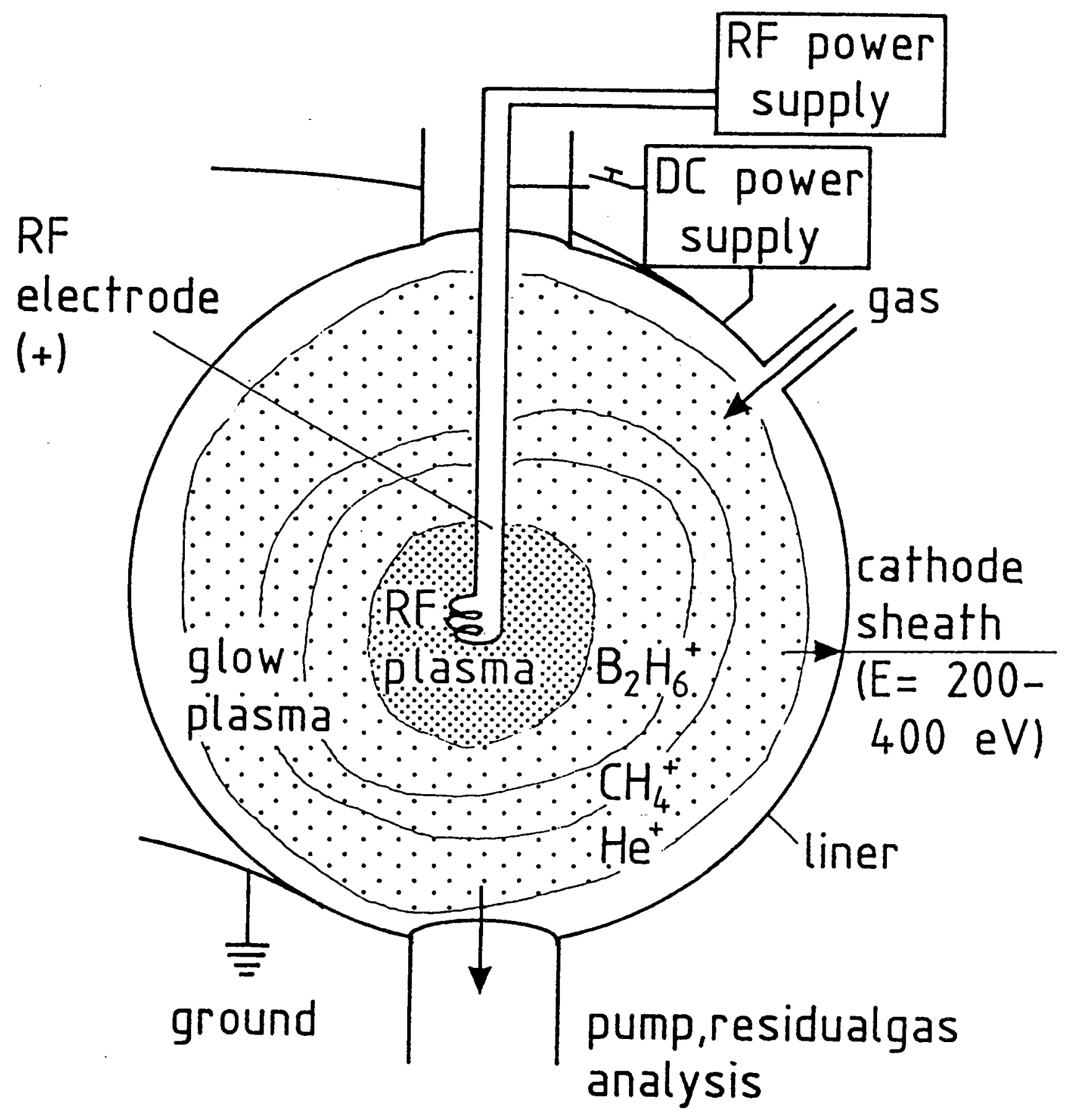


Fis. 4

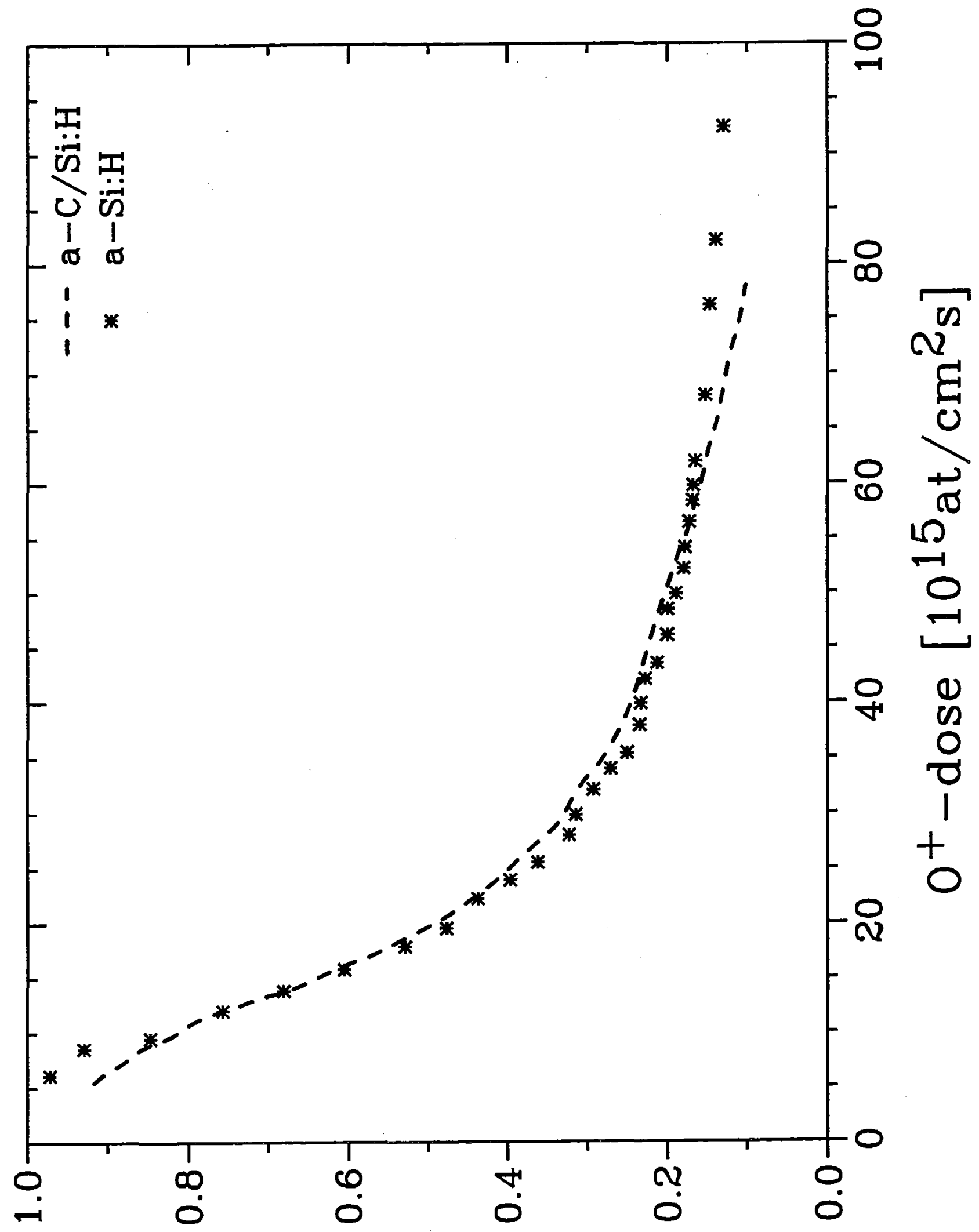

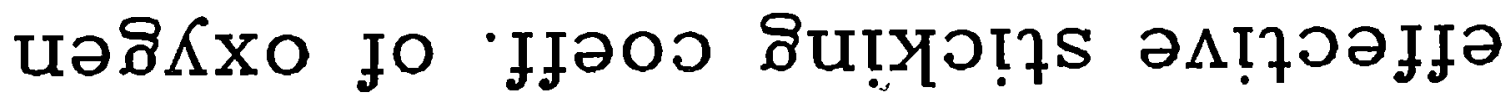




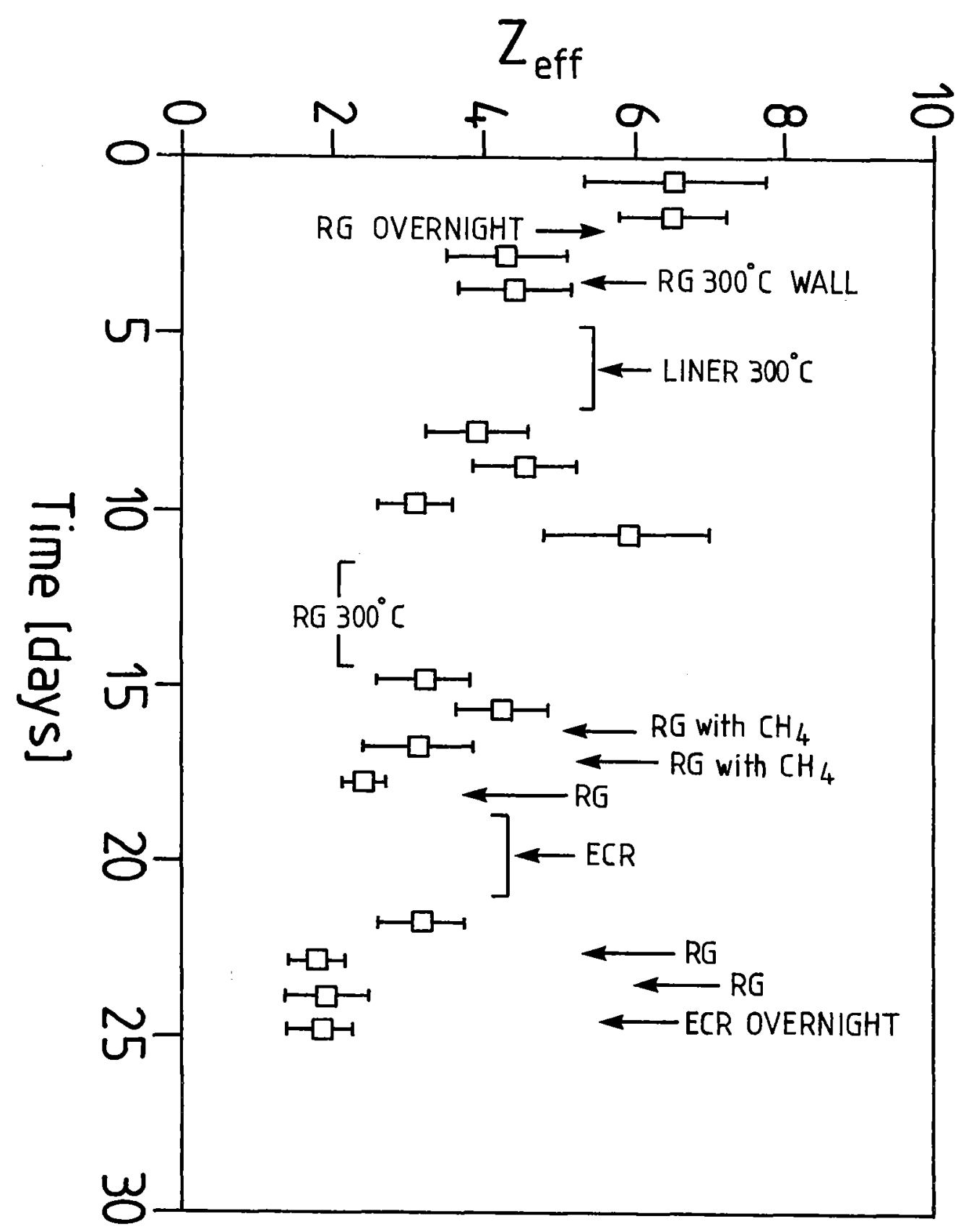


Fig. "

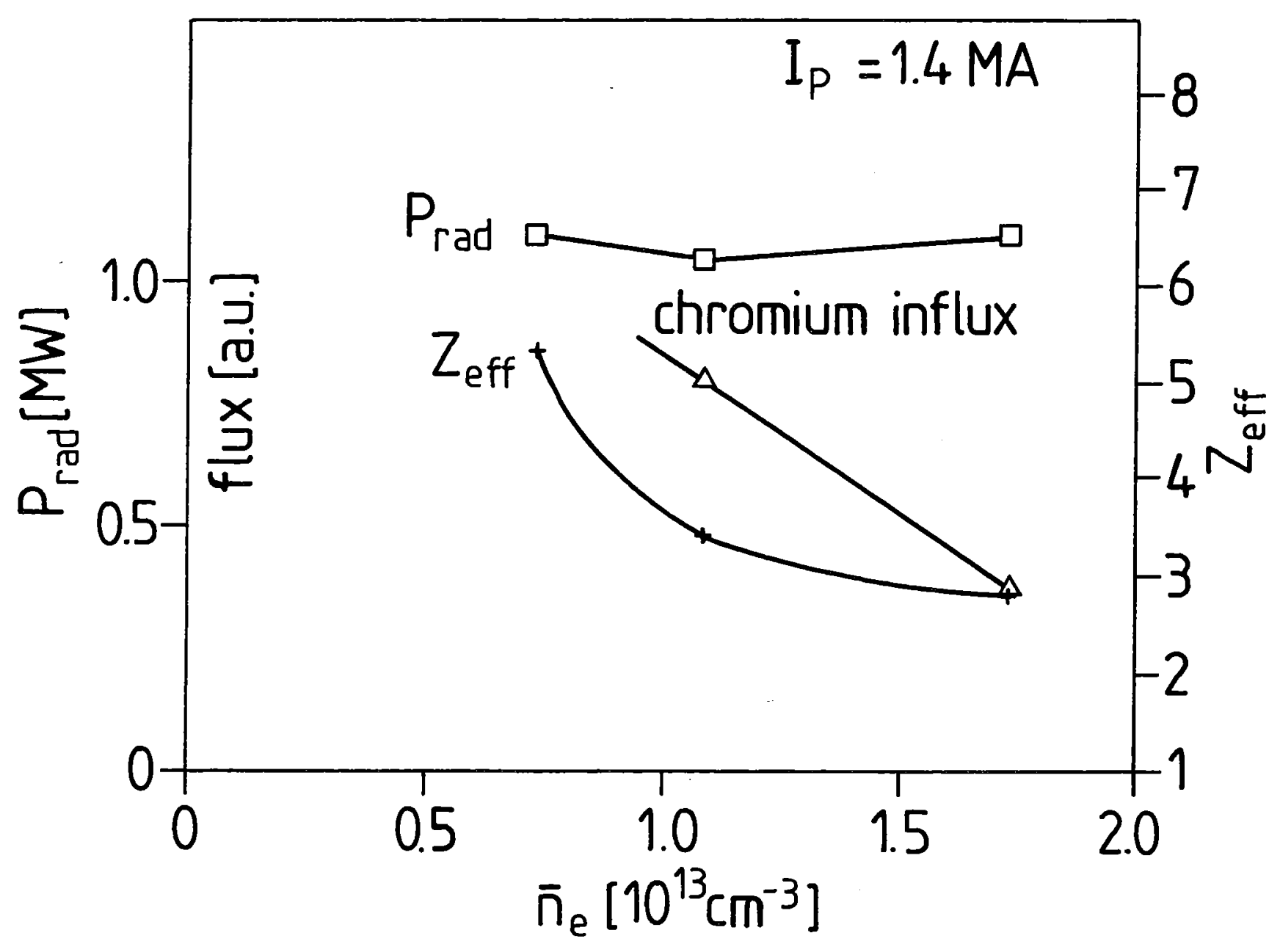




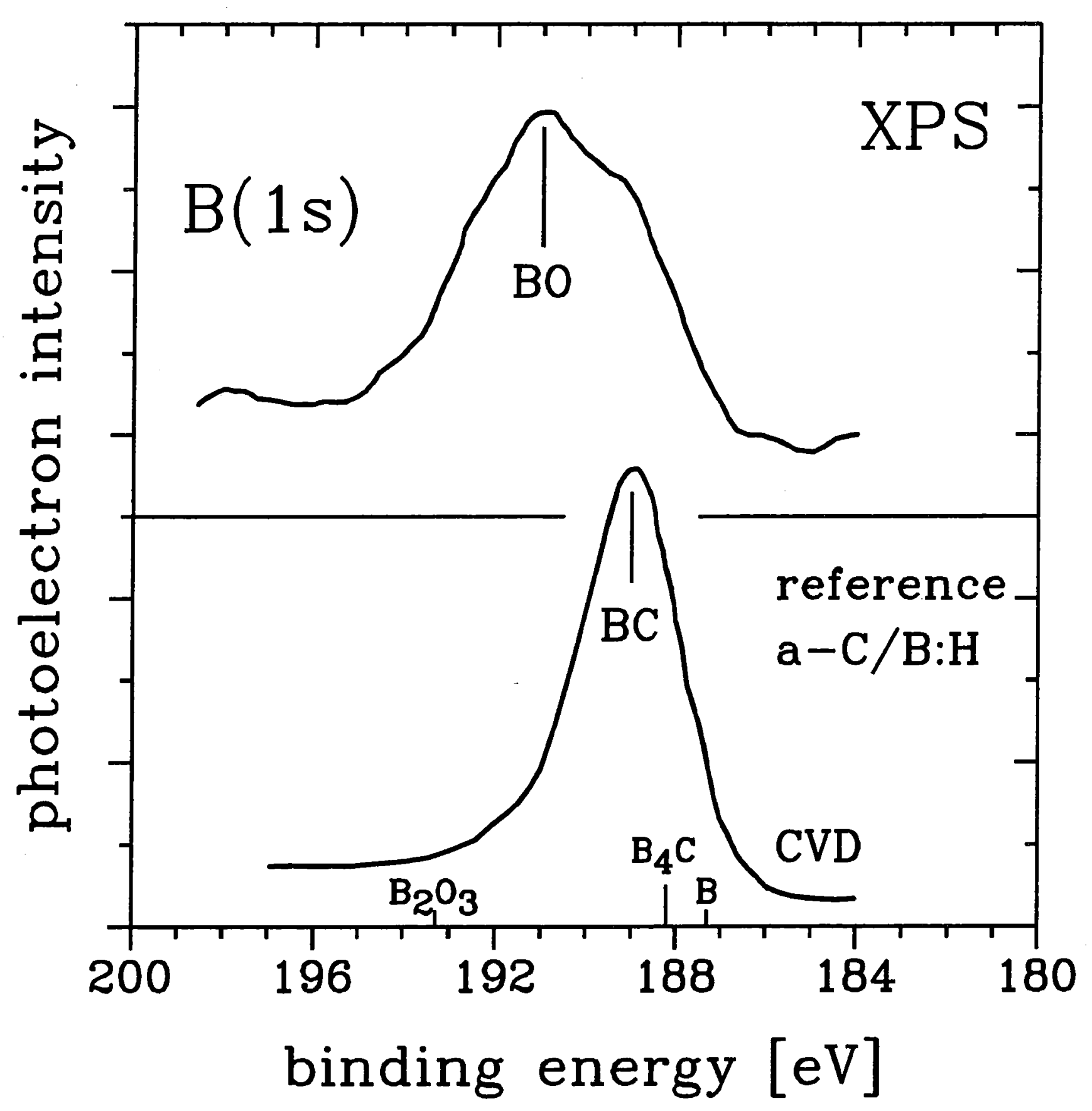


Fig. 10

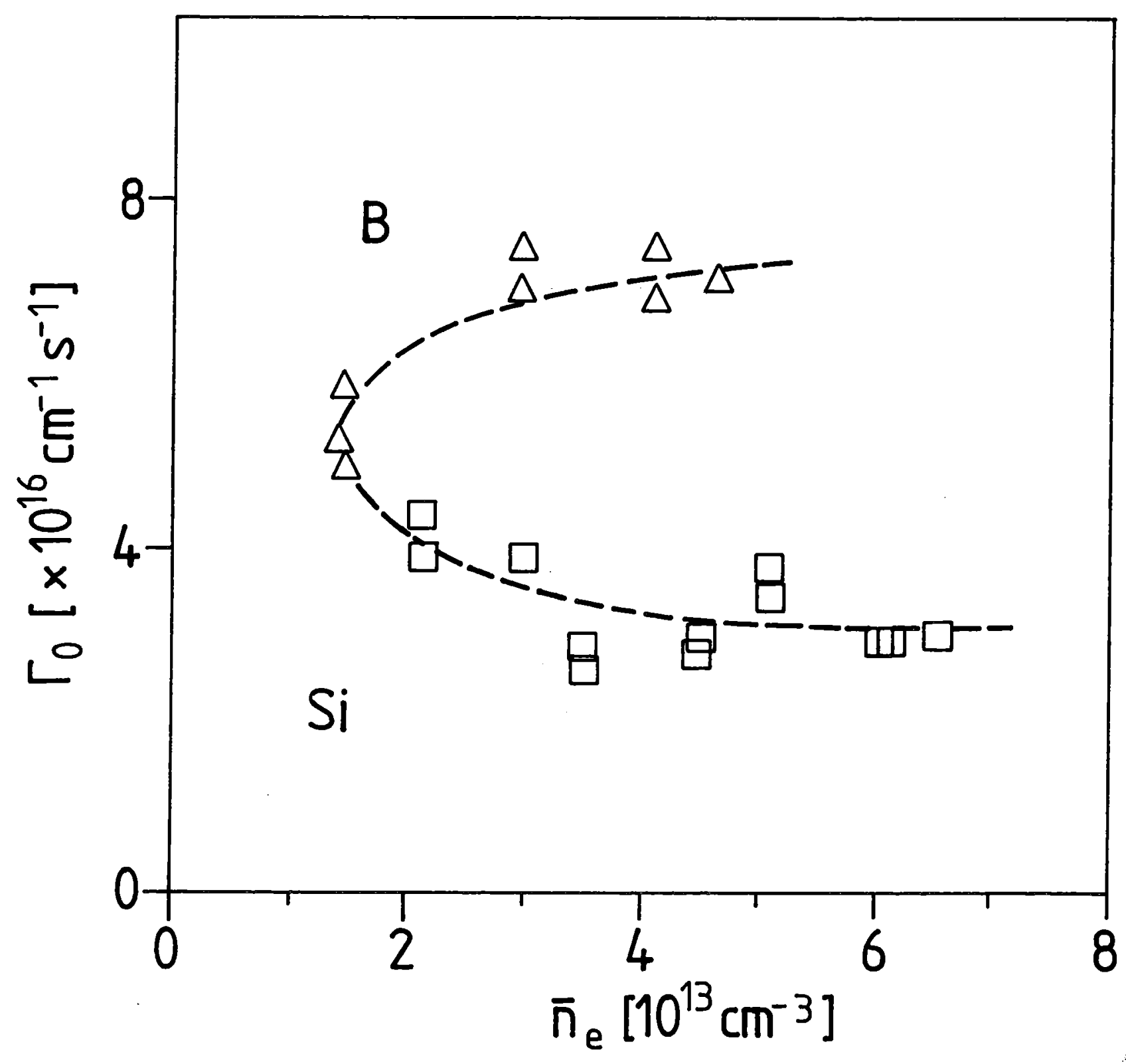




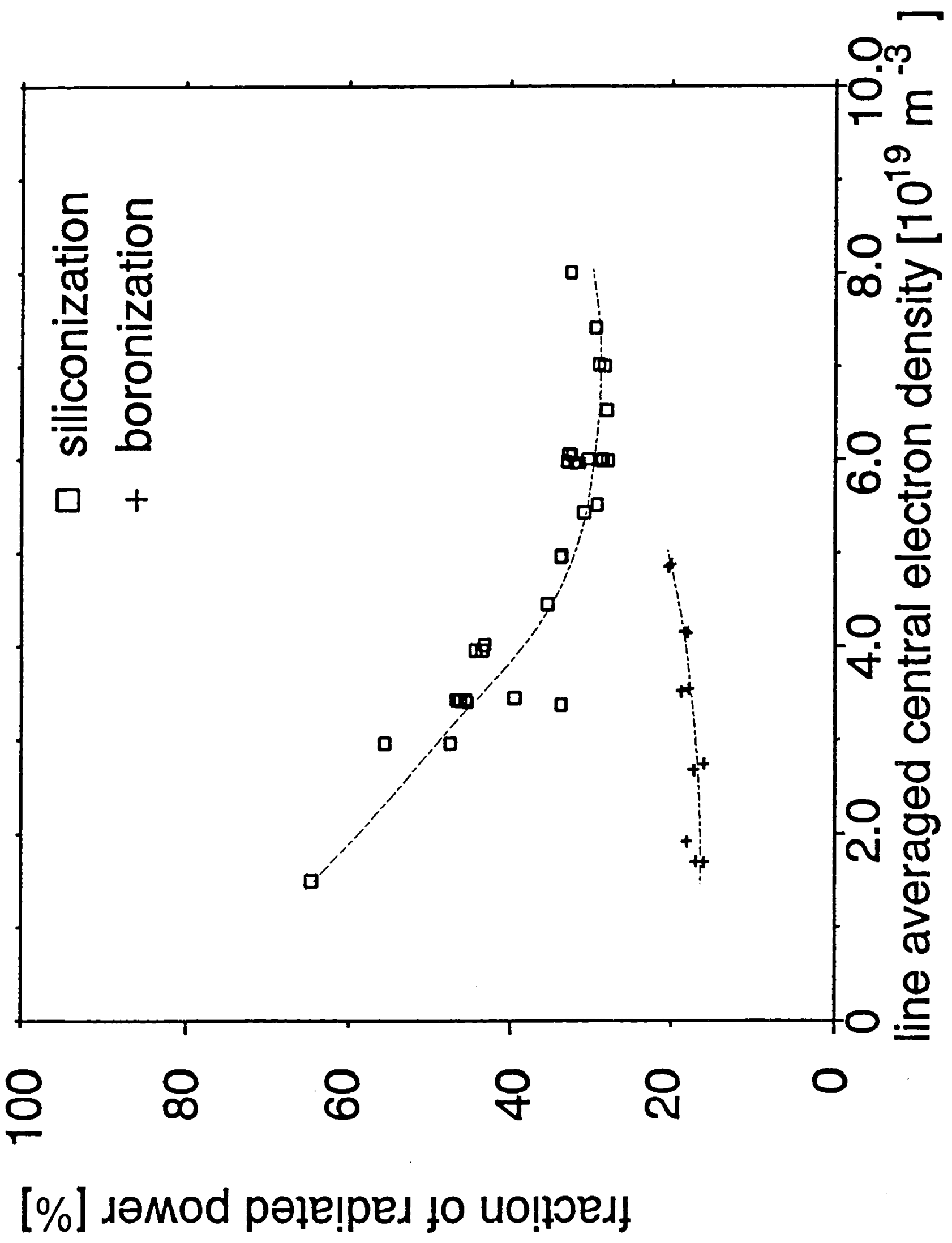




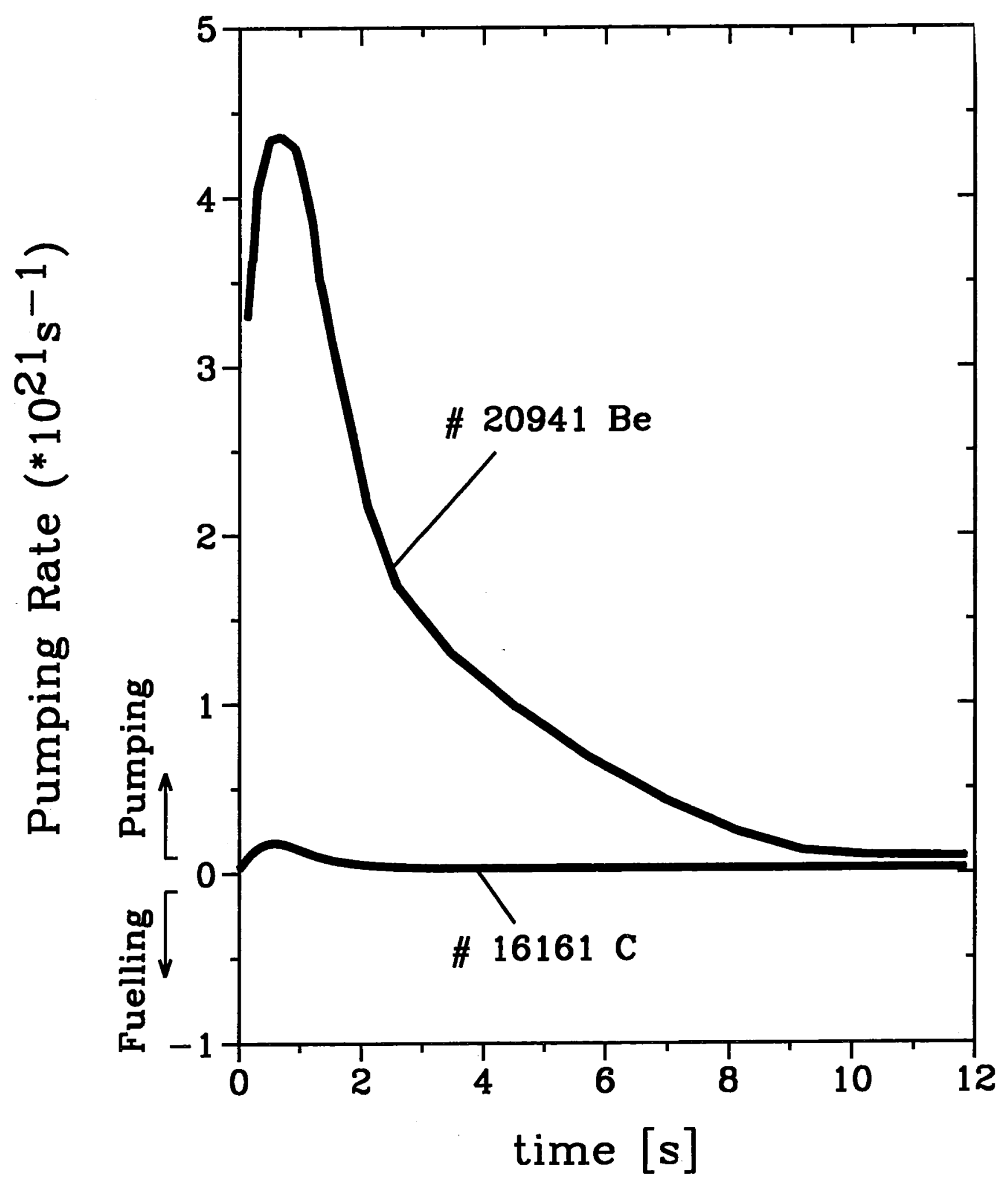


$F_{i j}, 2$
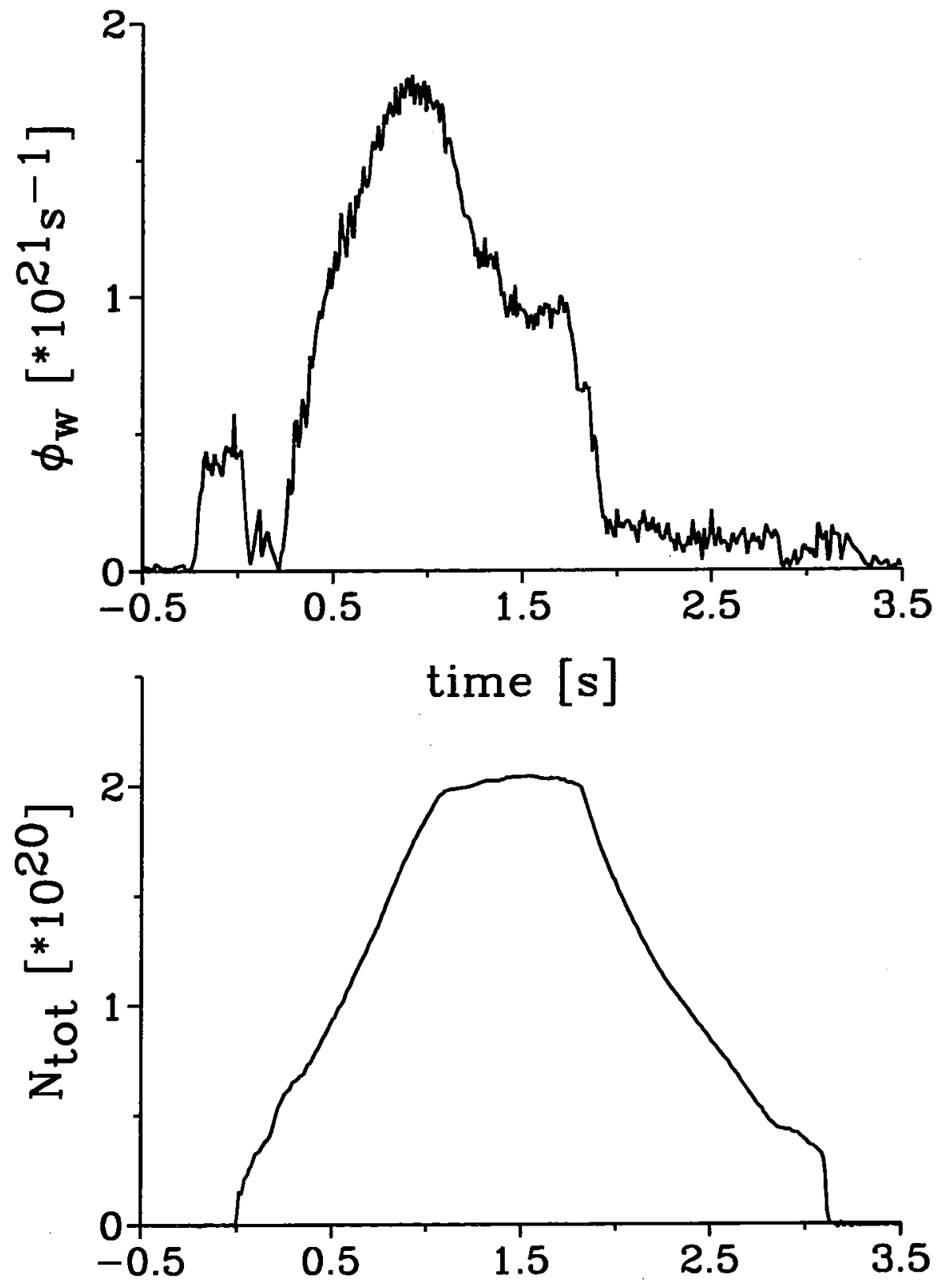

(a)

(b)

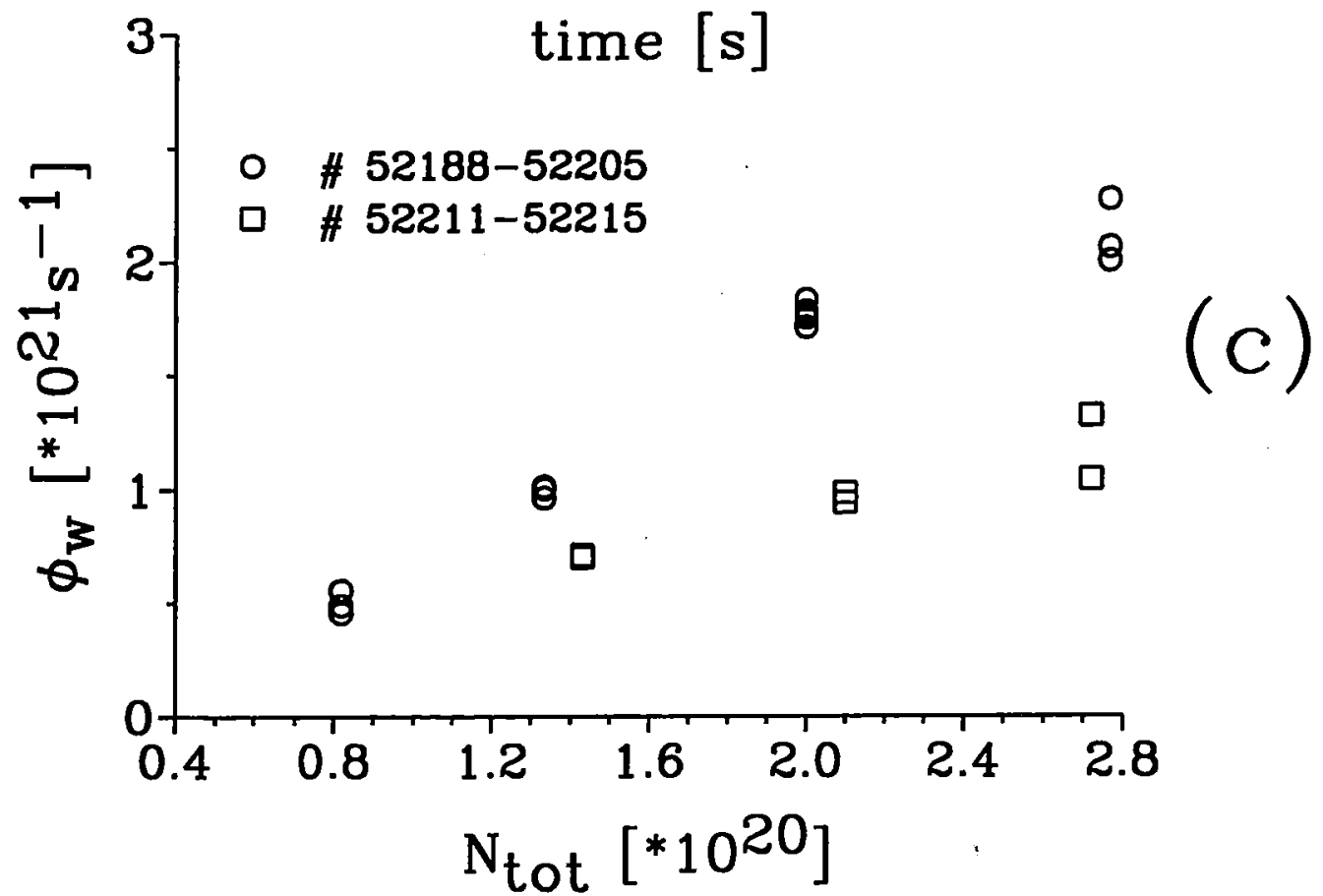




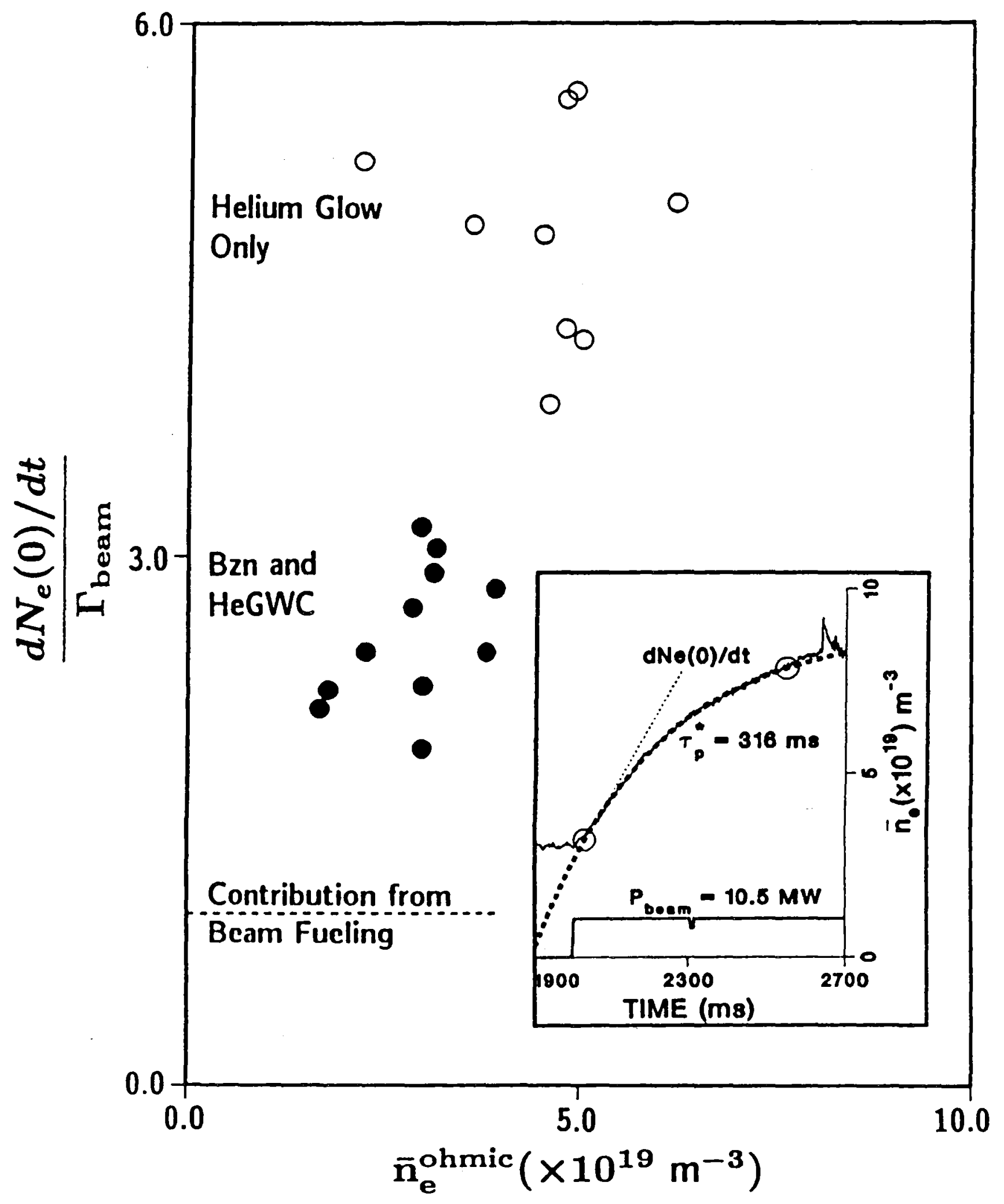




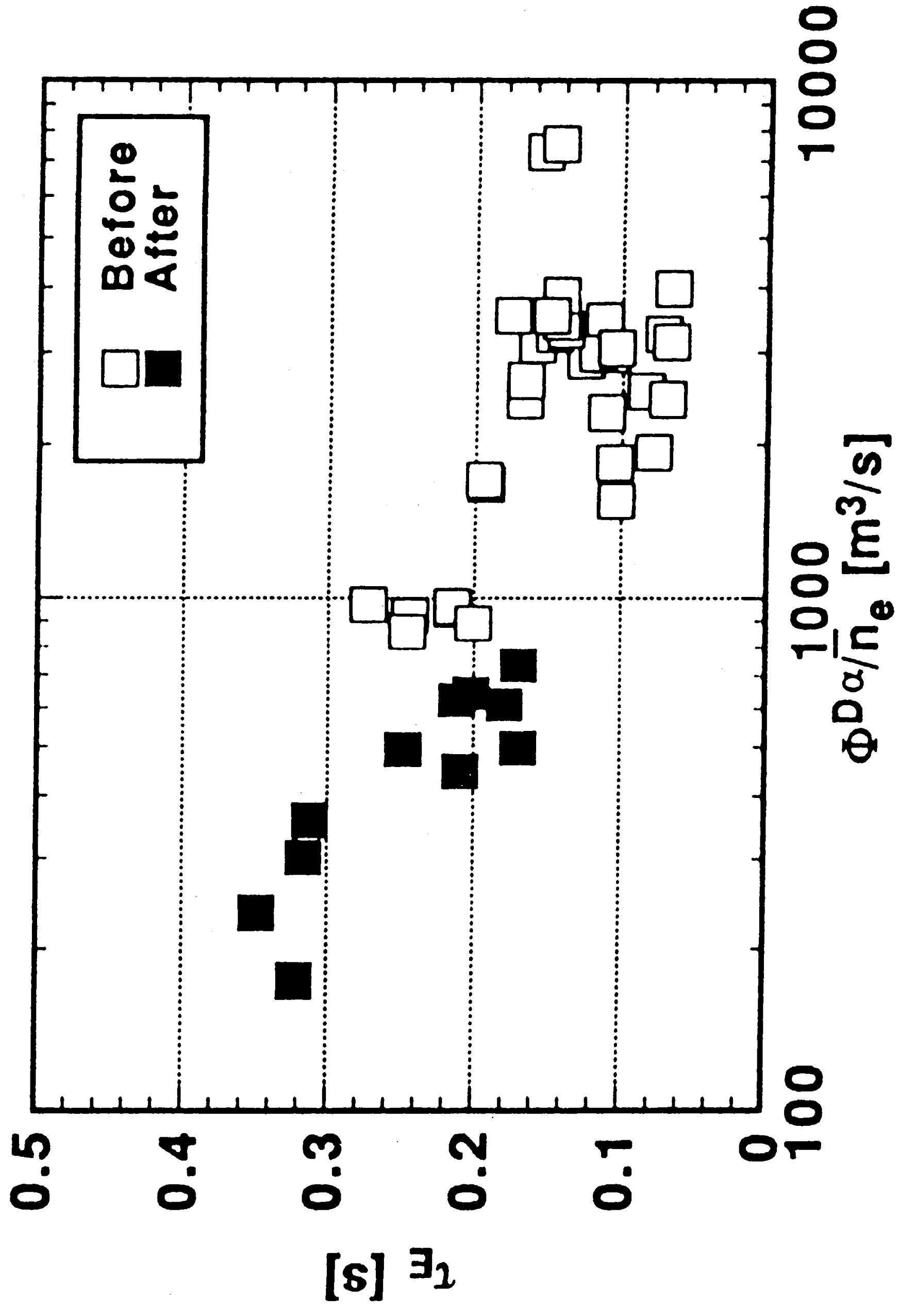


Fig. 24

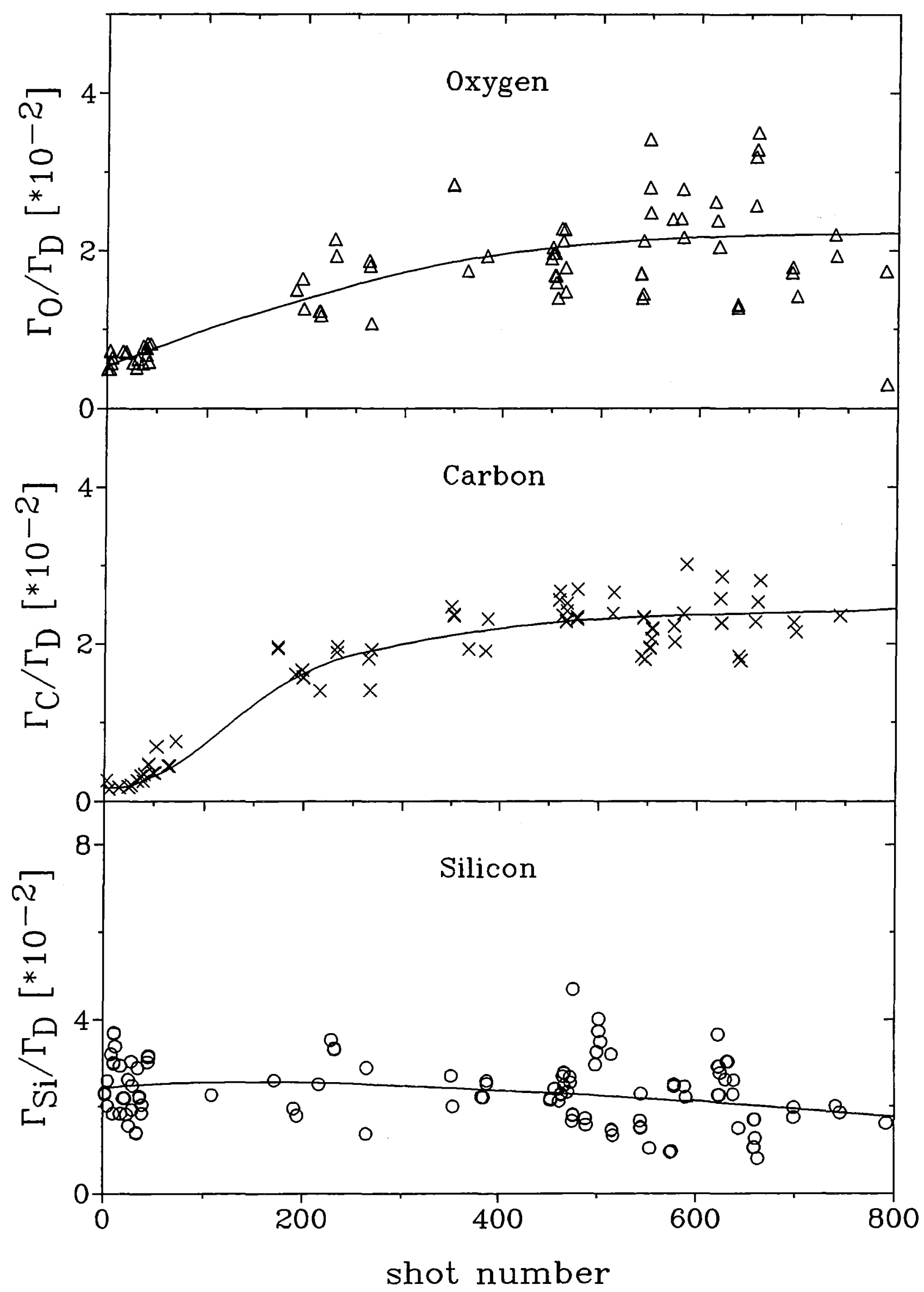


Fig 250

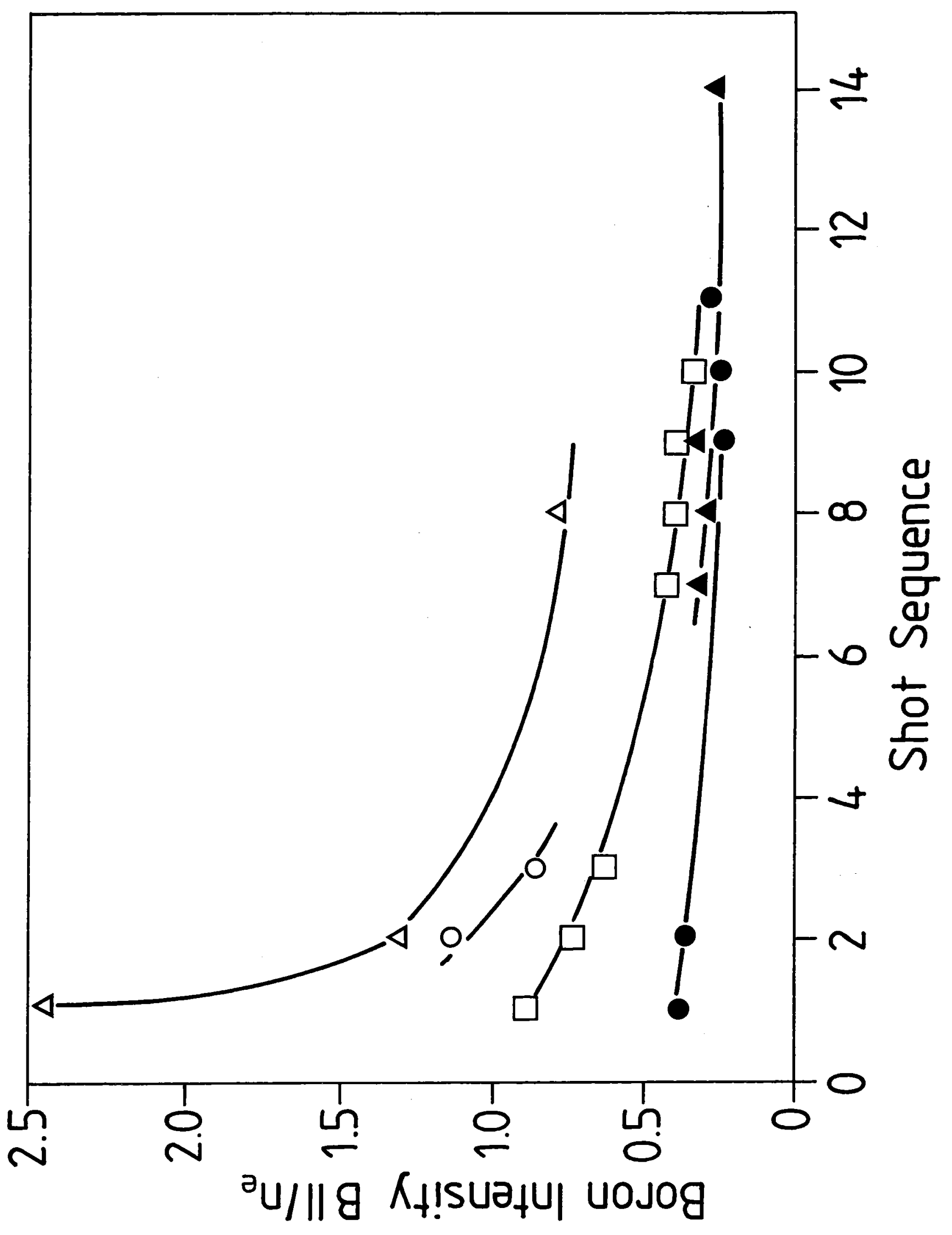




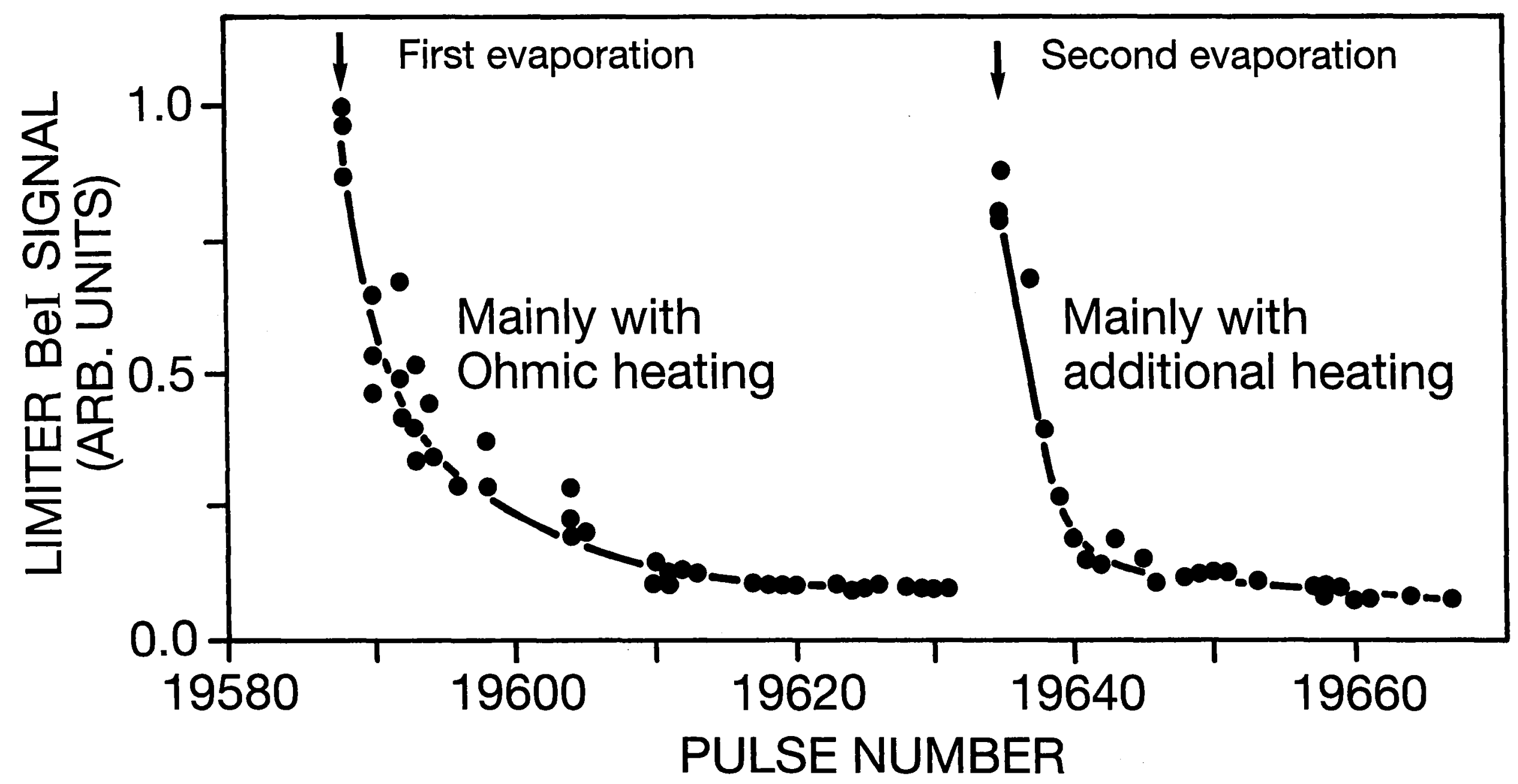


